\title{
The Gromov width of 4-dimensional tori
}

\author{
JANKO LATSCHEV \\ DUSA MCDUFF \\ FELIX SCHLENK
}

Let $\omega$ be any linear symplectic form on the 4-torus $T^{4}$. We show that in all cases $\left(T^{4}, \omega\right)$ can be fully filled by one symplectic ball. If $\left(T^{4}, \omega\right)$ is not symplectomorphic to a product $T^{2}(\mu) \times T^{2}(\mu)$ of equal sized factors, then it can also be fully filled by any finite collection of balls provided only that their total volume is less than that of $\left(T^{4}, \omega\right)$.

57R17, 57R40; 32J27

\section{Introduction}

It has been known since Gromov's paper [16] that symplectic embedding questions lie at the heart of symplectic geometry. For instance, Gromov's nonsqueezing theorem implies that for every natural number $k$, there is no symplectically embedded ball in the product $S^{2}(k) \times S^{2}(1)$ of $2-$ spheres of areas $k$ and 1 that fills more than $\frac{1}{2 k}$ of the volume. In this paper we study symplectic embeddings of balls into 4-dimensional tori with linear symplectic forms. Our main result is that the only obstruction to symplectically embedding a 4-ball into such a manifold is the total volume.

Consider the open ball of capacity $a$,

$$
B^{2 n}(a)=\left\{\left.z \in \mathbb{C}^{n}\left|\pi \sum_{j=1}^{n}\right| z_{j}\right|^{2}<a\right\},
$$

in standard symplectic space $\left(\mathbb{R}^{2 n}, \omega_{0}\right)$, where $\omega_{0}=\sum_{j=1}^{n} d x_{j} \wedge d y_{j}$. The Gromov width of a $2 n$-dimensional symplectic manifold $(M, \omega)$, introduced in [16], is defined as

$$
c_{\mathrm{G}}(M, \omega)=\sup \left\{a \mid B^{2 n}(a) \text { symplectically embeds into }(M, \omega)\right\} .
$$

Computations and estimates of the Gromov width for various examples can be found in Biran [4; 5], Biran and Cieliebak [6], Gromov [16], Jiang [19], Karshon and Tolman 
[20], Lalonde and McDuff [22; 23], Lu [29], McDuff [32], McDuff and Polterovich [33], McDuff and Slimowitz [35] and Schlenk [36].

If the symplectic manifold $(M, \omega)$ has finite volume, an invariant equivalent to its Gromov width is the ball filling number

$$
p(M, \omega)=\sup \frac{\operatorname{Vol}\left(B^{2 n}(a)\right)}{\operatorname{Vol}(M, \omega)},
$$

where the supremum is taken over all balls $B^{2 n}(a)$ that symplectically embed into $(M, \omega)$, and where the volume is defined as $\frac{1}{n !} \int_{M} \omega^{n}$. Since $\operatorname{Vol}\left(B^{2 n}(a)\right)=\frac{a^{n}}{n !}$,

$$
p(M, \omega)=\frac{\left(c_{G}(M, \omega)\right)^{n}}{n ! \operatorname{Vol}(M, \omega)} .
$$

If $p(M, \omega)<1$ one says that there is a filling obstruction, while if $p(M, \omega)=1$ one says that $(M, \omega)$ admits a full filling by one ball. ${ }^{1}$ In this paper our main focus is the filling number of 4-tori with a linear symplectic form $\omega$, ie, those which can be identified with the quotient of $\mathbb{R}^{4}$, with its standard symplectic structure, by a suitable lattice $\Lambda$. We also study other related filling questions in which the ball is replaced by a disjoint union of balls.

Filling obstructions usually come from non-constant holomorphic spheres. In tori, however, there are no such spheres. One can thus believe that for tori there should be no filling obstructions. For the standard torus $T(1,1):=\mathbb{R}^{4} / \mathbb{Z}^{4}$, there is the obvious lower bound $p(T(1,1)) \geq \frac{1}{2}$ coming from the inclusion of the ball $B^{4}(1)$ into the polydisc $B^{2}(1) \times B^{2}(1)$; see also Figure 3 below. A better lower bound (namely $\left.p(T(1,1)) \geq \frac{8}{9}\right)$ comes from algebraic geometry; see (2-6) in Section 2.2. We give an explicit realization of this embedding in Example 4.9 below.

Our main result is:

Theorem 1.1 Every 4-dimensional linear symplectic torus admits a full filling by one ball; in other words, $p\left(T^{4}, \omega\right)=1$ for all linear $\omega$.

The symplectic (resp. Kähler) cone of a smooth oriented manifold $X$ is the set of cohomology classes $\alpha \in H^{2}(X ; \mathbb{R})$ that can be represented by a symplectic (resp. Kähler) form, where here we consider symplectic forms that are compatible with the given orientation on $X$ (resp. Kähler forms that are compatible with any complex structure giving this orientation). The symplectic cone $\mathcal{C}\left(T^{4}\right)$ of $T^{4}$ with a given

\footnotetext{
${ }^{1}$ Our ball filling number is called first packing number by other authors, and full fillings by one ball also go under the name of full packings by one ball. We refer to Section 7 for a discussion of full fillings versus very full fillings.
} 
orientation is $\left\{\alpha \in H^{2}\left(T^{4} ; \mathbb{R}\right) \mid \alpha^{2}>0\right\}$. Each such class has a linear representative. From Theorem 1.1, we get the following characterization of the symplectic cone of the 1-point blow-up of a given oriented torus $T^{4}$.

Corollary 1.2 Denoting by $E \in H_{2}(\tilde{X} ; \mathbb{Z})$ the homology class of the exceptional divisor (with some orientation) in $\tilde{X}=T^{4} \sharp \overline{\mathbb{C} P^{2}}$, the symplectic cone of $\tilde{X}$ is

$$
\mathcal{C}(\tilde{X})=\left\{\alpha \in H^{2}(\tilde{X} ; \mathbb{R}) \mid \alpha^{2}>0, \alpha(E) \neq 0\right\} .
$$

While there are many examples of non-Kähler symplectic manifolds, it is much harder to find Kähler manifolds for which the Kähler and symplectic cones differ. Some examples are given by Drăghici [12] and Li-Usher [28]. More recently, CasciniPanov [9] showed that the Kähler and symplectic cones differ for the one point blow-up of $T^{2} \times S^{2}$. With the help of Corollary 1.2 we obtain another simple example.

Corollary 1.3 Let $\tilde{X}$ be the blow-up $T^{4} \sharp \overline{\mathbb{C} P^{2}}$ of the 4-torus in one point. Then the symplectic cone of $\tilde{X}$ is strictly bigger than the Kähler cone.

Outline of the proof of Theorem 1.1 As we will see, for our purposes linear 4-tori divide into three classes: the standard torus $T(1,1)$ (and its rescalings), all other rational tori (in which $[\omega]$ is a multiple of a rational class), and irrational tori (in which the image of the homomorphism $\int \omega: H_{2}(M ; \mathbb{Z}) \rightarrow \mathbb{R}$ has rank at least 2 over $\left.\mathbb{Q}\right)$.

It turns out that every rational torus is (up to scaling) symplectomorphic to a product torus $T^{2}\left(d_{1}\right) \times T^{2}\left(d_{2}\right)$, where $d_{1}, d_{2} \in \mathbb{N}$ denote the areas of the two factors; see Lemma 2.1. Thus the family of product tori $T(1, \mu):=T^{2}(1) \times T^{2}(\mu)$ with $\mu \geq 1$ contains all rational tori, up to scaling. With this in mind, our proof proceeds as follows.

1 Linear algebra By a simple symplectic linear algebra argument, the tori $T(m, n)$ and $T(1, m n)$ are symplectomorphic for relatively prime integers $m, n$ (see Remark 2.2). Hence:

Lemma $1.4 p\left(T\left(\frac{m}{n}, 1\right)\right)=p(T(1, m n))$ for $m, n \in \mathbb{N}$ relatively prime.

2 Algebraic geometry Buchdahl [8] and Lamari [25] found a condition on a cohomology class $\alpha \in H^{1,1}(X ; \mathbb{R})$ on some complex surface $X$ that guarantees the existence of a Kähler representative of $\alpha$. We shall verify this condition on blow-ups of irrational tori to obtain:

Proposition 1.5 $p\left(T^{4}, \omega\right)=1$ for all irrational linear tori $\left(T^{4}, \omega\right)$. 
3 Full fillings of $T^{\mathbf{2}}(\mathbf{1}) \times S^{\mathbf{2}}(\boldsymbol{\mu})$ Denote by $S^{2}(\mu)$ the 2-sphere endowed with an area form of area $\mu$. Biran [4] proved that $T^{2}(1) \times S^{2}(\mu)$ can be fully packed by one ball provided that $\mu \geq 2$. We shall show that such an almost filling ball can be made to lie in the complement of a constant section $T^{2}(1) \times \mathrm{pt}$. Since the open disc bundle $T^{2}(1) \times D^{2}(\mu)=\left(T^{2}(1) \times S^{2}(\mu)\right) \backslash\left(T^{2}(1) \times \mathrm{pt}\right)$ symplectically embeds into $T^{2}(1) \times T^{2}(\mu)=T(1, \mu)$, we obtain:

Proposition $1.6 p(T(\mu, 1))=1$ for all $\mu \geq 2$.

Corollary $1.7 p(T(\mu, 1))=1$ for all $\mu \neq 1$.

Proof In view of Propositions 1.5 and 1.6 we need only consider $\mu \in(1,2) \cap \mathbb{Q}$. If we write $\mu=\frac{m}{n}$ with $m, n \in \mathbb{N}$ relatively prime, then $m>n \geq 2$, giving $m n \geq 6$. Hence Lemma 1.4 and Proposition 1.6 imply $p(T(\mu, 1))=p(T(1, m n))=1$.

4 A symplectic embedding construction The only case not covered by the above discussion is the standard product torus $T(1,1)$. To prove $p(T(1,1))=1$ we shall construct for each ball $B^{4}(a)$ of volume $a^{2} / 2<1$ an explicit symplectic embedding into $T(1,1)$. Fix $a<\sqrt{2}$. We start with an almost full embedding $B^{4}(a) \rightarrow \diamond \times \square$, where $\diamond \subset \mathbb{R}^{2}\left(x_{1}, x_{2}\right)$ is a diamond-shaped domain (see Figure 3(I) below), and $\square=(0,1)^{2} \subset \mathbb{R}^{2}\left(y_{1}, y_{2}\right)$. The main step is then to construct a symplectic embedding $\diamond \times \square \rightarrow \mathbb{R}^{4}$ with image $U$ such that the projection $\mathbb{R}^{4} \rightarrow T(1,1)=\mathbb{R}^{4} / \mathbb{Z}^{4}$ is injective on $U$.

The resulting embedding $B^{4}(a) \rightarrow T(1,1)$ uses all four homological directions of $T(1,1)$. This must be so. Indeed, assume that there exists an embedding $B^{4}(a) \rightarrow$ $T(1,1)$ that factors, for instance, as

$$
B^{4}(a) \stackrel{\psi}{\longrightarrow} T^{3}\left(x_{1}, y_{1}, x_{2}\right) \times(0,1) \rightarrow T(1,1)
$$

with $(0,1) \subset \mathbb{R}\left(y_{2}\right)$. It is easy to see that there exists a symplectic embedding $\rho$ of the annulus $T^{1}\left(x_{2}\right) \times(0,1)$ into $B^{2}(1) \subset \mathbb{R}^{2}\left(x_{2}, y_{2}\right)$. Composing $\psi$ with id $\times \rho$ we obtain a symplectic embedding of $B^{4}(a)$ into $T^{2}\left(x_{1}, y_{1}\right) \times B^{2}(1)$, which lifts to $\mathbb{R}^{2}\left(x_{1}, y_{1}\right) \times B^{2}(1)$. Hence $a \leq 1$ by the nonsqueezing theorem. A similar discussion applies to all sufficiently large balls in product tori $T(\mu, 1)$ with $1 \leq \mu<2$.

Remark 1.8 Parts of the above construction yield an explicit full filling by one ball of the 4-torus $T(\mu, 1)$ for all $\mu=2 m^{2} / n^{2}$ with $m, n$ relatively prime. Since the set of rational numbers $\mu$ of this form is dense in $\mathbb{R}_{>0}$, one is tempted to derive $p(T(1,1))=1$ from $p(T(\mu, 1))=1$ for $\mu>1$ by a limiting argument, or to derive 
$p(T(\mu, 1))=1$ for all $\mu \geq 1$ from the elementary explicit full fillings of $T(\mu, 1)$ for $\mu=2 m^{2} / n^{2}$. However, without further knowledge about the underlying embeddings, the function $\mu \mapsto p(T(\mu, 1))$ has no obvious continuity properties.

Filling by more than one ball The general ball packing problem for a symplectic 4-manifold $(M, \omega)$ is: Given a collection $\bar{B}^{4}\left(a_{1}\right), \ldots, \bar{B}^{4}\left(a_{k}\right)$ of closed balls, does there exist a symplectic embedding of $\bigsqcup_{j=1}^{k} \bar{B}^{4}\left(a_{j}\right)$ into $(M, \omega)$ ? Since symplectic embeddings are volume-preserving, a necessary condition is

$$
\operatorname{Vol}\left(\coprod_{j=1}^{k} \bar{B}^{4}\left(a_{j}\right)\right)<\operatorname{Vol}(M, \omega) .
$$

We prove that for all linear tori except possibly $T(1,1)$ this is the only condition.

Theorem 1.9 Assume that $\left(T^{4}, \omega\right)$ is a linear torus. Let $\bar{B}^{4}\left(a_{1}\right), \ldots, \bar{B}^{4}\left(a_{k}\right)$ be a collection of balls such that

$$
\operatorname{Vol}\left(\coprod_{j=1}^{k} \bar{B}^{4}\left(a_{j}\right)\right)<\operatorname{Vol}\left(T^{4}, \omega\right) .
$$

(i) If $\left(T^{4}, \omega\right)$ is not symplectomorphic to $T(\mu, \mu)$ for some $\mu>0$, there exists a symplectic embedding of $\coprod_{j=1}^{k} \bar{B}^{4}\left(a_{j}\right)$ into $\left(T^{4}, \omega\right)$.

(ii) If $\left(T^{4}, \omega\right)$ is symplectomorphic to $T(\mu, \mu)$ for some $\mu>0$, then $\coprod_{j=1}^{k} \bar{B}^{4}\left(a_{j}\right)$ symplectically embeds into $T(\mu, \mu)$ under the further restriction that $a_{j}<\mu$ for all $j$.

Notice that Theorem 1.9 generalizes Propositions 1.5 and 1.6. The extra condition in (ii) is presumably not needed, but the only way we can see to prove this would be by explicitly constructing suitable embeddings.

Other examples of manifolds for which the volume is the only obstruction to a symplectic embedding of a collection of balls were found by Biran in $[4 ; 5]$. Biran also proved in [5] that $T(1,1)$ can be fully packed with $k$ equal balls for any $k \geq 2 .^{2}$

\footnotetext{
${ }^{2}$ In fact, his argument also proves the claim in Theorem 1.9 concerning $T(1,1)$. His proof is much the same as ours in that he reduces the problem to packing some ruled 4-manifold. However, he considers the projectivization of a holomorphic line bundle of Chern class 2 over a genus 2 surface, while we use a trivial bundle over $T^{2}$. In both cases the spherical fibers have area 1.
} 
Remarks 1.10 (i) Our results may give the impression that symplectic embeddings of balls into 4-dimensional tori are as flexible as volume-preserving embeddings. This is far from true, as the following consideration from [5] shows: By our results above, the standard product torus $T(1,1)$ admits symplectic embeddings of the ball $B(a)$ and of the disjoint union of two equal size balls $B(b) \sqcup B(b)$ whenever there is no volume obstruction. However, as is well known, a symplectic embedding of $B(b) \sqcup B(b)$ into $B(a)$ covers at most half of the volume. Therefore, given symplectic embeddings $\varphi: B(a) \rightarrow T(1,1)$ and $\psi: B(b) \sqcup B(b) \rightarrow T(1,1)$ that cover more than half of the volume, it cannot be that the image of $\varphi$ contains the image of $\psi$. This "hidden rigidity" phenomenon for symplectic embeddings of balls into tori clearly does not exist for volume-preserving embeddings of balls into tori.

(ii) Another important invariant of a symplectic manifold $(M, \omega)$ is its Hofer-Zehnder capacity $c_{\mathrm{HZ}}(M, \omega)$, which is of dynamical nature. We refer to the books Hofer and Zehnder [17] and McDuff and Salamon [34] for the definition and elementary properties. The value of this capacity is unknown for product tori; in fact it is an outstanding problem to decide whether it is finite or infinite for product tori.

Our computations of the Gromov width $c_{\mathrm{G}}$ of tori give lower bounds for $c_{\mathrm{HZ}}$, because $c_{\mathrm{G}}(M, \omega) \leq c_{\mathrm{HZ}}(M, \omega)$ for all symplectic manifolds. These lower bounds are, however, weaker than the known ones. These come from the elementary inequality

$$
c_{\mathrm{HZ}}(M, \omega) \geq c_{\mathrm{HZ}}\left(P, \omega_{P}\right)+c_{\mathrm{HZ}}\left(Q, \omega_{Q}\right),
$$

holding for all products $(M, \omega)=\left(P \times Q, \omega_{P} \oplus \omega_{Q}\right)$ of closed symplectic manifolds, together with the fact that the Hofer-Zehnder capacity of a 2-dimensional connected symplectic manifold equals its area. To be explicit, our main theorem implies that $c_{\mathrm{HZ}}(T(1,1)) \geq c_{\mathrm{G}}(T(1,1))=\sqrt{2}$, while it is known that $c_{\mathrm{HZ}}(T(1,1)) \geq 1+1=2$.

The paper is organized as follows. In Section 2 we review the lower bounds for the ball filling number of 4-dimensional symplectic tori coming from known computations of Seshadri constants. Section 3.1 contains a proof of Theorem 1.9 in the irrational case. This proof is based on the construction in Section 3.2 of symplectic tori with no holomorphic curves. In Section 3.3 we prove Theorem 1.9 for product tori $T(1, \mu)$, $\mu \geq 1$, under the condition that $\min \left\{a_{j}, b_{j}\right\}<\mu$ for all $j$. In Section 4 we explain the embedding construction that we use in Section 5 to prove $p(T(1,1))=1$, completing the proof of Theorem 1.1. In Section 6 we prove Corollaries 1.2 and 1.3, and in Section 7 we state some open problems related to filling tori.

Acknowledgements This work has its origin in discussions between the authors and Dietmar Salamon at the Edifest 2010, and we would like to thank ETH Zürich and its 
FIM for the stimulating atmosphere during the conference. We also thank Paul Biran, Dietmar Salamon and Sewa Shevchishin for fruitful discussions. Finally, we thank the referee for a careful reading and helpful comments on the exposition.

Dusa McDuff is partially supported by NSF grant number DMS 0905191. Felix Schlenk is partially supported by SNF grant number 200021-125352/1.

\section{Relations to algebraic geometry}

In this section, we review the implications of some results in algebraic geometry for the Gromov radius of 4-dimensional symplectic tori, and also of some higher-dimensional ones.

\subsection{Basics}

Before discussing the complex geometry of tori, we recall a classical result.

Lemma 2.1 Suppose $\omega$ is a linear symplectic form on a torus $T=\mathbb{R}^{2 n} / \Lambda$ with integral cohomology class. Then $(T, \omega)$ is symplectomorphic to a product of $2-$ dimensional tori

$$
T^{2}\left(d_{1}\right) \times \cdots \times T^{2}\left(d_{n}\right)
$$

with symplectic areas $d_{j}>0$ satisfying $d_{j} \mid d_{j+1}$ for all $j=1, \ldots, n-1$. Moreover, the sequence $d_{1}\left|d_{2}\right| \cdots \mid d_{n}$ is uniquely determined by $\Lambda$.

Remark 2.2 It follows that a 4-dimensional product torus $T(m, n)=T^{2}(m) \times T^{2}(n)$ with integer areas $m$ and $n$ is symplectomorphic to $T(g, \ell)$, where $g=\operatorname{gcd}(m, n)$, $\ell=\operatorname{lcm}(m, n)$.

Proof of Lemma 2.1 Since $\omega$ is linear, it lifts to a linear symplectic form on $\mathbb{R}^{2 n}$, which we again denote by $\omega$. The fact that it represents an integral cohomology class on $T$ is equivalent to the fact that it takes integer values when restricted to $\Lambda \times \Lambda$. Denote by $d_{1} \in \mathbb{Z}$ the positive generator of this image subgroup, and choose $e_{1}, f_{1} \in \Lambda$ with $\omega\left(e_{1}, f_{1}\right)=d_{1}$.

Every lattice point $\lambda \in \Lambda$ can be written as

$$
\lambda=\frac{\omega\left(\lambda, f_{1}\right)}{d_{1}} e_{1}+\frac{\omega\left(e_{1}, \lambda\right)}{d_{1}} f_{1}+\lambda^{\prime},
$$

where the coefficients of $e_{1}$ and $f_{1}$ are integers by the choice of $d_{1}$, and where $\lambda^{\prime} \in \Lambda$ is $\omega$-orthogonal to both $e_{1}$ and $f_{1}$. In other words, $\Lambda=\operatorname{span}_{\mathbb{Z}}\left(e_{1}, f_{1}\right) \oplus \Lambda^{\prime}$ for some 
lower-dimensional sublattice $\Lambda^{\prime} \subset \Lambda$. Now repeat the argument with $\Lambda^{\prime}$ in place of $\Lambda$, noting that the image of $\omega$ when restricted to $\Lambda^{\prime} \times \Lambda^{\prime}$ must be a subgroup of $d_{1} \mathbb{Z} \subset \mathbb{Z}$. This finishes the proof in $n$ steps.

To prove the uniqueness of the sequence $d_{1}|\cdots| d_{n}$ for a given torus $T=\mathbb{R}^{2 n} / \Lambda$, note that since $\omega$ is non-degenerate and integral, it gives rise to an embedding $\phi: \Lambda \rightarrow$ $\operatorname{Hom}(\Lambda, \mathbb{Z})$, namely $\phi\left(\lambda_{1}\right)\left(\lambda_{2}\right)=\omega\left(\lambda_{1}, \lambda_{2}\right)$. Now the $d_{j}$ are the torsion coefficients of the finitely generated abelian group $\operatorname{Hom}(\Lambda ; \mathbb{Z}) / \operatorname{Im} \phi$, which are well-known to be invariants of this group.

Complex tori are often defined as the quotients of $\mathbb{C}^{n}$ by some cocompact lattice $\Lambda \cong \mathbb{Z}^{2 n}$. In dimension 4, the Enriques-Kodaira classification of compact complex surfaces implies that every complex manifold diffeomorphic to $T^{4}$ is biholomorphic to such a model. In higher dimensions, this is still true if the complex structure is compatible with a Kähler form, but false in general (for examples, see eg Catanese, Oguiso and Peternell [10] and references therein).

Conversely, the standard symplectic form on $\mathbb{C}^{n}$ descends to a Kähler form on any quotient $\mathbb{C}^{n} / \Lambda$, so every complex torus admits a compatible Kähler structure whose symplectic form is translation invariant.

\subsection{Seshadri constants of tori}

Here we review some results described by Lazarsfeld in [27, Chapter 5], which do not seem to be widely known among symplectic geometers. For an irreducible projective variety $X$ and a point $x \in X$ we denote by

$$
\pi: \tilde{X} \rightarrow X
$$

the blow-up of $X$ at $x$, with exceptional divisor $\Sigma \subset \tilde{X}$. Recall that a line bundle $L$ on $X$ is called nef if for every irreducible curve $C \subset X$ one has $\int_{C} c_{1}(L) \geq 0$.

Definition 2.3 (cf [27, Definition 5.1.1]) Suppose $L$ is a nef line bundle on $X$. The Seshadri constant of $(X, L)$ at $x \in X$ is defined to be the real number

$$
\varepsilon(L ; x):=\max \left\{\varepsilon \geq 0 \mid \int_{\widetilde{C}} \pi^{*}\left(c_{1} L\right)-\varepsilon \Sigma \cdot \widetilde{C} \geq 0 \text { for all curves } \widetilde{C} \subset \tilde{X}\right\}
$$

It is clear that $\varepsilon(L, x)$ is always nonnegative, and in fact one has the alternative description (cf [27, Proposition 5.1.5])

$$
\varepsilon(L ; x)=\inf _{x \in C \subset X} \frac{\int_{C} c_{1}(L)}{\text { mult }_{x} C},
$$


where the infimum is taken over all irreducible curves $C \subset X$ passing through $x$, and mult $_{x} C \in \mathbb{N}$ denotes the multiplicity of $C$ at $x$. This shows that one can obtain upper bounds on $\varepsilon(L ; x)$ from specific curves passing through $x \in X$.

From the symplectic point of view, we are particularly interested in the case when $X$ is a smooth projective variety, and $L$ is an ample line bundle. Then one can choose a Kähler form $\omega_{L}$ representing $c_{1}(L)$. Since the space of symplectic forms in a fixed cohomology class that are compatible with a fixed (almost) complex structure is contractible, any two such forms are symplectically isotopic.

Now there is a strong relationship between symplectic embeddings of balls and symplectic blow-up, which was first described by McDuff [30], and McDuff and Polterovich [33]: An embedding of a closed symplectic ball $\bar{B}(a)$ of capacity $a$ into a given symplectic manifold $X$ gives rise to a symplectic form on the topological blow-up $\pi: \tilde{X} \rightarrow X$ whose cohomology class is given by $\pi^{*}[\omega]-a \operatorname{PD}[\Sigma]$, where $\Sigma \subset \tilde{X}$ is the exceptional divisor, and $\operatorname{PD}[\Sigma]$ denotes the Poincaré dual of $[\Sigma]$. Conversely, given a tame symplectic form on the complex blow-up $(\tilde{X}, \tilde{J})$ in a class $\pi^{*} \alpha-a \operatorname{PD}[\Sigma]$, one can find a symplectically embedded ball $\bar{B}(a)$ in $(X, \omega)$ with $[\omega]=\alpha \in H^{2}(X ; \mathbb{R})$; see [33, Corollary 2.1.D].

As pointed out in [27, Theorem 5.1.22], this discussion then leads to the following result, which is a direct consequence of [33, Corollary 2.1.D]:

Proposition 2.4 For fixed $X$ and $L$ as above, denote by

$$
\varepsilon(X, L):=\max _{x \in X} \varepsilon(L ; x) .
$$

Then the Gromov width of $\left(X, \omega_{L}\right)$, defined in (1-1), satisfies

$$
c_{G}\left(X, \omega_{L}\right) \geq \varepsilon(X, L) .
$$

By the relation (1-2), this estimate is equivalent to

$$
p\left(X, \omega_{L}\right) \geq \frac{(\varepsilon(X, L))^{n}}{n ! \operatorname{Vol}\left(X, \omega_{L}\right)} .
$$

The proof of Proposition 2.4 is based on the fact that when $\varepsilon(X ; L)>0$, then the pullback $\tilde{L}$ of $L$ to the blow-up $\tilde{X}$ is ample, so that $c_{1}(\tilde{L})$ has a Kähler representative.

Remark 2.5 The same blow-up argument also shows that the capacity of the largest symplectically and holomorphically embedded ball in the Kähler manifold $\left(X, \omega_{L}\right)$ bounds the Seshadri constant $\varepsilon(X, L)$ from below (for details, see [27, Proposition 5.3.17]). 
In what follows, we will study the family of symplectic product tori $T^{2}(1) \times T^{2}(d)$, where $d \in \mathbb{Z}$. By Lemma 2.1, up to rescaling this class contains all symplectic 4-tori whose symplectic form is linear and has a rational cohomology class. Now suppose that $(T, \omega)$ is such a symplectic torus, and choose a translation-invariant compatible complex structure $J$, so that $(T, J, \omega)$ is a Kähler manifold. If $L$ is the complex line bundle on $(T, J)$ with first Chern class $[\omega]$, then $L$ is ample. Complex tori admitting such a line bundle are called abelian varieties, and the line bundle or its first Chern class is called a polarization. Note that, conversely, the first Chern class of any ample line bundle $L$ on some complex torus can be represented by a translation-invariant rational symplectic form $\omega_{L}$, and so all abelian varieties arise as above.

The sequence of integers $\left(d_{1}, \ldots, d_{n}\right)$ for $\left(T, \omega_{L}\right)$ appearing in Lemma 2.1 is called the type of the polarization, and the polarization is called principal and often denoted by $\Theta$ if it is of type $(1, \ldots, 1)$, ie, it corresponds to the standard symplectic product torus.

Since translations act transitively on any abelian variety $A$, the Seshadri constants for abelian varieties do not depend on the choice of the point $x \in A$. One has the general bounds

$$
d_{1} \leq \varepsilon\left(A^{n}, L\right) \leq\left(n ! d_{1} \cdots d_{n}\right)^{\frac{1}{n}}
$$

for an ample line bundle $L$ of type $\left(d_{1}, \ldots, d_{n}\right)$. The upper bound follows from the estimate (2-3); see also [27, Proposition 5.1.9]. For the lower bound, recall from Lemma 2.1 that $d_{1}\left|d_{2}\right| \cdots \mid d_{n}$, and consider the ample line bundle $L^{\prime}$ of type $\left(1, d_{2} / d_{1}, \ldots, d_{n} / d_{1}\right)$. By [27, Example 5.3.10], one has $\varepsilon\left(A, L^{\prime}\right) \geq 1$, and therefore $\varepsilon(A, L) \geq d_{1}$. Similarly, the symplectic embedding of the ball of capacity $d_{1}$ into the polydisc $B^{2}\left(d_{1}\right) \times \cdots \times B^{2}\left(d_{n}\right) \subset A$ gives the same lower bound $d_{1}$ for the Gromov width of $\left(A, \omega_{L}\right)$.

The best lower bounds on Seshadri constants for abelian varieties of a given type seem to come from irreducible ones, ie, those which cannot be written as a product of lower-dimensional complex tori. Here we list the known results, according to [27, Remark 5.3.12].

First, to get a bound on the ball filling number of $T(1,1)$, according to the discussion above we need to consider principally polarized abelian surfaces $\left(A^{2}, \Theta\right)$. Steffens [37, Propositions 2 and 3] has shown that in this case

$$
\varepsilon\left(A^{2}, \Theta\right) \leq \frac{4}{3},
$$


with equality if $A$ is irreducible. Together with the estimate (2-3) we obtain the lower bound

$$
p(T(1,1)) \geq \frac{8}{9}
$$

For tori of type $(1, d)$ one can get lower bounds from non-principal polarizations of abelian surfaces $\left(A^{2}, L\right)$. Indeed, it is known from the work of Steffens [37, Proposition 1] that if $2 d$ is a perfect square, then there are abelian surfaces with a polarization $L$ of type $(1, d)$ and

$$
\varepsilon\left(A^{2}, L\right)=\sqrt{2 d}
$$

which is optimal since it equals the volume bound in (2-4). This immediately implies

$$
p(T(1, d))=1 \quad \text { if } 2 d \text { is a perfect square. }
$$

We will describe explicit examples of such full fillings by one symplectic ball in Section 4.3. The identities (2-8) and Remark 2.2 imply that $p(T(\mu, 1))=1$ for all $\mu=2 m^{2} / n^{2}$ with $m, n$ relatively prime integers.

On the other hand, when $2 d$ is not a perfect square, then Bauer and Szemberg [1] have shown that

$$
\varepsilon\left(A^{2}, L\right) \leq 2 d \frac{k_{0}}{\ell_{0}}=\sqrt{2 d} \cdot \sqrt{\frac{2 d k_{0}^{2}}{2 d k_{0}^{2}+1}},
$$

where $\left(k_{0}, \ell_{0}\right)$ is the smallest solution in positive integers of Pell's equation

$$
\ell^{2}-2 d k^{2}=1
$$

(There always exists such a solution, as was first shown by Lagrange [21].) Moreover, by a result of Bauer [2], equality holds whenever positive multiples of $L$ are the only ample line bundles on $A$. Since complex structures $J$ with this property exist for all symplectic types $(1, d)$, this gives the best constant for use in Proposition 2.4. For $d \leq 30$, the relevant solutions to Pell's equation have been tabulated in [2, page 572], and we give their translation in terms of the lower bound on the ball filling numbers

$$
p(T(1, d)) \geq \frac{\varepsilon^{2}}{2 d}=\frac{\ell_{0}^{2}-1}{\ell_{0}^{2}}
$$

in the following table. 


\begin{tabular}{|c|ccc|c|ccc|c|ccc|}
\hline$d$ & $k_{0}$ & $\ell_{0}$ & $\frac{\varepsilon^{2}}{2 d}$ & $d$ & $k_{0}$ & $\ell_{0}$ & $\frac{\varepsilon^{2}}{2 d}$ & $d$ & $k_{0}$ & $\ell_{0}$ & $\frac{\varepsilon^{2}}{2 d}$ \\
\hline 1 & 2 & 3 & $\frac{8}{9}$ & 11 & 42 & 197 & $\frac{38808}{38809}$ & 21 & 2 & 13 & $\frac{168}{169}$ \\
2 & & & 1 & 12 & 1 & 5 & $\frac{24}{25}$ & 22 & 30 & 199 & $\frac{39600}{39601}$ \\
3 & 2 & 5 & $\frac{24}{25}$ & 13 & 10 & 51 & $\frac{2600}{2601}$ & 23 & 3588 & 24335 & $\frac{592192224}{592192225}$ \\
4 & 1 & 3 & $\frac{8}{9}$ & 14 & 24 & 127 & $\frac{16128}{16129}$ & 24 & 1 & 7 & $\frac{48}{49}$ \\
5 & 6 & 19 & $\frac{360}{361}$ & 15 & 2 & 11 & $\frac{120}{121}$ & 25 & 14 & 99 & $\frac{9800}{9801}$ \\
6 & 2 & 7 & $\frac{48}{49}$ & 16 & 3 & 17 & $\frac{288}{289}$ & 26 & 90 & 649 & $\frac{421200}{421201}$ \\
7 & 4 & 15 & $\frac{224}{225}$ & 17 & 6 & 35 & $\frac{1224}{1225}$ & 27 & 66 & 485 & $\frac{235224}{235225}$ \\
8 & & & 1 & 18 & & & 1 & 28 & 2 & 15 & $\frac{224}{225}$ \\
9 & 4 & 17 & $\frac{288}{289}$ & 19 & 6 & 37 & $\frac{1368}{1369}$ & 29 & 2574 & 19605 & $\frac{384356024}{384356025}$ \\
10 & 2 & 9 & $\frac{80}{81}$ & 20 & 3 & 19 & $\frac{360}{361}$ & 30 & 4 & 31 & $\frac{960}{961}$ \\
\hline
\end{tabular}

\subsection{Seshadri estimates for higher-dimensional tori}

One well-studied class of principally polarized abelian varieties of arbitrary dimension are the Jacobians of curves (cf eg [7, Chapter 11]). Here we just recall that the Jacobian of a complex curve $C$ is the complex torus

$$
J C:=\operatorname{Hom}\left(\Omega^{1,0}, \mathbb{C}\right) / H_{1}(C ; \mathbb{Z}),
$$

where $\Omega^{1,0}$ denotes the complex vector space of holomorphic 1 -forms, and the embedding $H_{1}(C ; \mathbb{Z}) \subset \operatorname{Hom}\left(\Omega^{1,0}, \mathbb{C}\right)$ is given by integration over cycles. The complex dimension of $J C$ equals the genus of $C$, and the principal polarization is derived from the natural symplectic structure on $H_{1}(C ; \mathbb{Z}) \otimes \mathbb{R}$, which is given by the intersection product.

In complex dimension $n=3$, Bauer and Szemberg [3] have shown that a principally polarized abelian variety $\left(A^{3}, \Theta\right)$ has $\varepsilon(\Theta)=\frac{3}{2}$ if $A$ is the Jacobian of a hyperelliptic curve of genus 3 and

$$
\varepsilon\left(A^{3}, \Theta\right)=\frac{12}{7}
$$

otherwise. (A complex curve is called hyperelliptic if it admits a double branched cover to $\mathbb{C} P^{1}$.) Hence $p(T(1,1,1)) \geq \frac{288}{343}$.

In complex dimension $n=4$, Debarre [11] has shown that for the Jacobian $A^{4}=J C$ of a non-hyperelliptic curve of genus 4 one has

$$
\varepsilon\left(A^{4}, \Theta\right)=2 \text {. }
$$


Hence $p(T(1,1,1,1)) \geq \frac{2}{3}$.

For high dimensions, Jacobians appear to give very poor lower bounds for use in Proposition 2.4. However, Lazarsfeld [26] combined the work of McDuff and Polterovich [33] with work of Buser and Sarnak on minimal period lengths to deduce that there exist principally polarized abelian varieties $\left(A^{n}, \Theta\right)$ of complex dimension $n$ with

$$
\varepsilon\left(A^{n}, \Theta\right) \geq \frac{1}{4}(2 n !)^{\frac{1}{n}} .
$$

Bauer has generalized this, showing that there exist polarized abelian varieties $\left(A^{n}, L\right)$ of arbitrary type $\left(d_{1}, \ldots, d_{n}\right)$ with

$$
\varepsilon\left(A^{n}, L\right) \geq \frac{1}{4}\left(2 n ! d_{1} \ldots d_{n}\right)^{\frac{1}{n}} .
$$

While this is only a factor of less than 4 away from the upper bound of (2-4), the volume fraction filled by the symplectic ball predicted from this lower bound is $2\left(\frac{1}{4}\right)^{n}$, and hence tends exponentially to zero as $n \rightarrow \infty$.

\section{Proof of Theorem 1.9}

\subsection{Irrational case}

We will use the following result of Buchdahl [8] and Lamari [25]:

Theorem 3.1 $[8 ; 25]$ Let $(X, J)$ be a compact complex surface. A cohomology class $\alpha \in H^{1,1}(X ; \mathbb{R})$ admits a Kähler representative compatible with the complex structure $J$ if $\alpha \cup \alpha>0, \alpha \cup[\rho]>0$ for some positive closed $(1,1)$-form $\rho$ on $X$ and $\alpha \cdot[D]>0$ for every effective divisor $D \subset X .^{3}$

In symplectic language, the last condition means that the class $\alpha$ should integrate positively over every compact holomorphic curve in $X$.

Our argument is based on the following result, whose proof is deferred until the next subsection.

Proposition 3.2 Any irrational linear symplectic form $\omega$ on $T^{4}$ may be identified with a Kähler form on a torus $T=\mathbb{C}^{2} / \Lambda$ that has no nonconstant compact holomorphic curves.

\footnotetext{
${ }^{3}$ Notice that the form $\rho$ is a Kähler form. Therefore, Buchdahl's condition that the first Betti number of $X$ is even is automatic.
} 
Proposition 3.3 Theorem 1.9 holds for irrational tori.

Proof We must show that any disjoint union $\bigsqcup_{j=1}^{k} \bar{B}^{4}\left(a_{j}\right)$ of balls symplectically embeds into $\left(T^{4}, \omega\right)$ provided only that the volume constraint is satisfied. By Proposition 3.2 there is a symplectomorphism from $\left(T^{4}, \omega\right)$ to $\left(T, \omega_{J}\right)$ where $\left(T, J, \omega_{J}\right)$ is the Kähler torus found in Proposition 3.2. Let $\left(T_{k}, J_{k}\right)$ be the complex blow-up of $T$ at $k$ generic points $x_{j}$, and consider the cohomology class

$$
\alpha:=\pi^{*}\left(\left[\omega_{J}\right]\right)-\sum_{j=1}^{k} a_{j} \operatorname{PD}\left[\Sigma_{j}\right],
$$

where $\pi: T_{k} \rightarrow T$ is the blow-down map and the $\Sigma_{j}$ are the exceptional divisors. Since the complex structure $J$ and the symplectic form $\omega_{J}$ on $T$ have constant coefficients, we find for each $j$ an $\varepsilon_{j}>0$ and an embedding $\psi^{j}: B^{4}\left(\varepsilon_{j}\right) \rightarrow T$ with $\psi^{j}(0)=x_{j}$ that is holomorphic and symplectic (ie, $\psi_{*}^{j} J_{\text {can }}=J$ and $\psi_{*}^{j} \omega_{\text {can }}=\omega$ ); see [34, Exercise 2.52(iii)]. Take $\varepsilon>0$ such that $\varepsilon \leq \varepsilon_{j}$ for each $j=1, \ldots, k$ and such that

$$
\int_{T} \omega_{J} \wedge \omega_{J}>\sum_{j=1}^{k} \varepsilon a_{j} .
$$

Let $\rho$ be the Kähler form on $T_{k}$ corresponding to the blow-up defined by the $k$ embeddings $\psi^{j}: B^{4}(\varepsilon) \rightarrow T$. Then

$$
[\rho]=\pi^{*}\left(\left[\omega_{J}\right]\right)-\sum_{j=1}^{k} \varepsilon \operatorname{PD}\left[\Sigma_{j}\right],
$$

whence $\alpha \cup[\rho]>0$. Furthermore, the volume condition gives $\alpha^{2}>0$, and the only compact holomorphic curves in $\left(T_{k}, J_{k}\right)$ are the $\Sigma_{j}$. The criterion of Buchdahl and Lamari thus holds for the class $\alpha$. Therefore there is a Kähler form $\tau$ on $\left(T_{k}, J_{k}\right)$ in class $\alpha$. Blowing down the form $\tau$ we obtain a symplectic form $\eta$ on $T$ in class $\left[\omega_{J}\right]$, and disjoint symplectically embedded balls $\bar{B}^{4}\left(a_{1}\right), \ldots, \bar{B}^{4}\left(a_{k}\right)$ in $(T, \eta)$. It remains to show that $\eta$ is isotopic to $\omega_{J}$. Then Moser's argument shows that $(T, \eta)$ and $\left(T, \omega_{J}\right)$ are symplectomorphic. Hence the balls $\bigsqcup_{j} \bar{B}^{4}\left(a_{j}\right)$ also symplectically embed into $\left(T, \omega_{J}\right)$.

To find an isotopy from $\eta$ to $\omega_{J}$, we consider for each $s \in(0,1]$ the cohomology class

$$
\alpha_{s}:=\pi^{*}\left(\left[\omega_{J}\right]\right)-\sum_{j=1}^{k} s a_{j} \operatorname{PD}\left[\Sigma_{j}\right],
$$


and repeat the above construction to find Kähler forms $\tau_{s}$ on $\left(T_{k}, J_{k}\right)$ in class $\alpha_{s}$, $s \in(0,1]$. Moreover, choose $s_{0}>0$ such that $s_{0} a_{j}<\varepsilon$ for all $j$, and for $s \in\left(0, s_{0}\right)$ let $\rho_{s}$ be the Kähler form on $T_{k}$ corresponding to the blow-up defined by the $k$ embeddings $\psi^{j}: B^{4}\left(s_{0} a_{j}\right) \rightarrow T$. Denote by $\Omega\left(J_{k},\left\{\Sigma_{j}\right\}\right)$ the space of $J_{k}$-tame symplectic forms on $T_{k}$ that restrict to symplectic forms on the $\Sigma_{j}$. Then each form $\tau_{s}, s \in(0,1]$, and each form $\rho_{s}, s \in\left(0, s_{0}\right)$, belongs to $\Omega\left(J_{k},\left\{\Sigma_{j}\right\}\right)$. Since the space of forms in $\Omega\left(J_{k},\left\{\Sigma_{j}\right\}\right)$ in a given cohomology class is convex, we can alter the collection $\left\{\tau_{s}\right\}$ to a smooth family $\left\{\tau_{s}^{\prime}\right\}$ of forms in $\Omega\left(J_{k},\left\{\Sigma_{j}\right\}\right)$ such that $\tau_{s}^{\prime}$ is cohomologous to $\tau_{s}$, such that $\tau_{s}^{\prime}=\rho_{s}$ for $s \in\left(0, s_{0} / 2\right)$, and such that $\tau_{1}^{\prime}=\tau_{1}$. Now blow down the forms $\tau_{s}^{\prime}$ to obtain a smooth family $\eta_{s}$ of symplectic forms on $T$ in class $\left[\omega_{J}\right]$. By construction, $\eta_{1}=\eta$ and $\eta_{s}=\omega_{J}$ for $s \in\left(0, s_{0} / 2\right)$, as required.

Remark 3.4 The only property of irrational linear symplectic forms on $T^{4}$ that we used here was the existence of a compatible complex structure $J$ such that $H^{2}(T ; \mathbb{Z}) \cap$ $H^{1,1}(T ; \mathbb{C})$ vanishes, ie, such that the Picard number of $(T, J)$ vanishes. According to the Enriques-Kodaira classification, there is exactly one other class of compact Kähler surfaces with this property, namely K3-surfaces with Picard number 0. Repeating the above proof, we find that the conclusions of Theorem 1.9 also hold true for all Kähler forms on K3-surfaces for which a compatible complex structure has vanishing Picard number.

\subsection{Irrational tori with no curves}

We now prove Proposition 3.2.

We begin by finding complex tori with no nonconstant compact holomorphic curves. Consider $\mathbb{C}^{2}=\mathbb{C} e_{1} \oplus \mathbb{C} e_{2}$ and denote by $e_{1}, e_{2}, e_{3}=\sqrt{-1} e_{1}, e_{4}=\sqrt{-1} e_{2}$ the standard real basis. Choose real numbers $p, q, r, s$ such that

$$
p, q, r, s \text { are rationally independent, and } p s-q r \text { is irrational. }
$$

Consider the quotient of $\mathbb{C}^{2}$ by the lattice $\Lambda_{P}$ spanned by

$$
\lambda_{1}=e_{1}, \quad \lambda_{2}=e_{2}, \quad \lambda_{3}=p e_{3}+q e_{4}, \quad \lambda_{4}=r e_{3}+s e_{4} .
$$

The following result is extracted from in Elencwajg and Forster [14, Appendix]. We repeat it here for the sake of completeness.

Lemma 3.5 Under the assumption (3-1), the torus $T=\mathbb{C}^{2} / \Lambda_{P}$ contains no nonconstant compact holomorphic curves. 
Proof A nonconstant compact holomorphic curve would represent a nonzero class in $H_{1,1}(T ; \mathbb{C}) \cap H_{2}(T ; \mathbb{Z})$. By duality it thus suffices to prove that $H^{2}(T ; \mathbb{Z}) \cap$ $H^{1,1}(T ; \mathbb{C})=\{0\}$. Write the complex coordinates of $\mathbb{C}^{2}$ as $z_{j}=x_{j}+\sqrt{-1} y_{j}$. Since every class in $H^{2}(T ; \mathbb{C})$ can be represented by a form with constant coefficients, every class in $H^{1,1}(T ; \mathbb{C})$ has a representative of the form

$$
\begin{aligned}
\omega=x d x_{1} \wedge d y_{1}+y d x_{2} \wedge d y_{2}+u\left(d x_{1} \wedge d y_{2}+d x_{2} \wedge d y_{1}\right) \\
+v\left(d x_{1} \wedge d x_{2}+d y_{1} \wedge d y_{2}\right)
\end{aligned}
$$

where the coefficients $x, y, u, v$ are complex constants. The class $[\omega]$ will be integral if and only if the coefficients of the matrix

$$
\omega\left(\lambda_{i}, \lambda_{j}\right)=\left(\begin{array}{cccc}
0 & v & p x+q u & r x+s u \\
-v & 0 & p u+q y & r u+s y \\
-(p x+q u) & -(p u+q y) & 0 & v(p s-q r) \\
-(r x+s u) & -(r u+s y) & -v(p s-q r) & 0
\end{array}\right)
$$

are integers, ie, if

(i) $v, v(p s-q r) \in \mathbb{Z}$,

(ii) $p x+q u, r x+s u, p u+q y, r u+s y \in \mathbb{Z}$.

Since we have chosen $p s-q r$ irrational, the two conditions (i) imply $v=0$. Assume that $x, y, u$ fulfill the conditions (ii). We then find $n_{1}, n_{2}, n_{3}, n_{4} \in \mathbb{Z}$ with

$$
\begin{aligned}
p x+q u & =n_{1}, & p u+q y & =n_{3}, \\
r x+s u & =n_{2}, & r u+s y & =n_{4} .
\end{aligned}
$$

We can eliminate $x$ and $y$ from the above equations and obtain

$$
\begin{aligned}
& (p s-q r) u=-n_{1} r+n_{2} p, \\
& (p s-q r) u=n_{3} s-n_{4} q,
\end{aligned}
$$

which implies $-n_{1} r+n_{2} p-n_{3} s+n_{4} q=0$. Since we have chosen $p, q, r, s$ to be rationally independent, it follows that $n_{1}, n_{2}, n_{3}, n_{4}$ must vanish. Hence $u$ and therefore also $x$ and $y$ vanish. We conclude that $\omega=0$, as we wanted to show.

Now we start with a torus $T^{4}=\mathbb{R}^{4} / \Lambda$ with a linear symplectic form $\omega$ representing an irrational cohomology class. Given an integral basis $\lambda_{1}, \ldots, \lambda_{4}$ for $\Lambda$, the symplectic form $\omega$ can be represented by a matrix $B=\left(b_{i j}\right)$, where $b_{i j}=\omega\left(\lambda_{i}, \lambda_{j}\right)$. We denote by $\lambda_{1}^{*}, \ldots, \lambda_{4}^{*}$ the basis dual to $\lambda_{1}, \ldots, \lambda_{4}$, and we may assume that the ordering has been chosen such that $\omega \wedge \omega$ is a positive multiple of $\lambda_{1}^{*} \wedge \lambda_{3}^{*} \wedge \lambda_{2}^{*} \wedge \lambda_{4}^{*}$. 
Lemma 3.6 In the situation just described, after changing the basis of $\mathbb{R}^{4}$ by an element of $\mathrm{SL}(4, \mathbb{Z})$, we may represent $\omega$ by a matrix $B^{\prime}$, where

(i) the entries $b_{12}^{\prime}, b_{34}^{\prime}$ either both vanish or they are rationally independent and positive, and

(ii) the vector $\left(b_{13}^{\prime}, b_{14}^{\prime}, b_{23}^{\prime}, b_{24}^{\prime}\right)$ is not a multiple of a rational vector.

Proof Suppose first that there is a permutation $i_{1}, \ldots, i_{4}$ of $\{1, \ldots, 4\}$ so that $b_{i_{1} i_{2}}=$ $b_{i_{3} i_{4}}=0$. Then we can change basis (preserving orientation) so that $b_{12}^{\prime}=b_{34}^{\prime}=0$. Condition (ii) is then automatic since $\omega$ is irrational.

In all other cases we can permute the basis (preserving orientation) so that $b_{12} \neq 0$, $b_{34} \neq 0$, and so that at least two of the elements $b_{12}, b_{34}, b_{1 j}$ are rationally independent, where $j=3$ or 4 . If condition (i) is not satisfied, we change basis by replacing $\lambda_{2}^{*}$ by $\lambda_{2}^{*}+k \lambda_{j}^{*}$ and leaving the other elements fixed. Then $b_{12}$ changes to $b_{12}^{\prime}=b_{12}+k b_{1 j}$ and $b_{34}^{\prime}=b_{34}$, so that for suitable $k \in \mathbb{Z}$ we may assume that $b_{12}^{\prime}, b_{34}^{\prime}$ are rationally independent and of the same sign. If they are both negative, we can change their signs by interchanging $\lambda_{1}$ and $\lambda_{2}$ and interchanging $\lambda_{3}$ and $\lambda_{4}$. This achieves (i).

If (ii) does not hold, we may assume that the vector $\beta=\left(b_{13}, b_{14}, b_{23}, b_{24}\right)$ does not vanish, because otherwise by a permutation we could have arranged the situation with $b_{12}^{\prime}=b_{34}^{\prime}=0$ from the beginning of the proof, and, as observed there, (ii) is automatic in that case.

So one of the entries of $\beta$ must be nonzero and hence rationally independent of either $b_{12}$ or $b_{34}$. We will consider the case that $b_{13}$ and $b_{12}$ are rationally independent, the other cases being treated in a similar fashion. Now we change basis, replacing $\lambda_{4}^{*}$ by $\lambda_{4}^{*}+k \lambda_{1}^{*}$ and leaving the other elements fixed. Then $b_{i j}^{\prime}=b_{i j}$ if $i, j \neq 4$, while $b_{i 4}^{\prime}=b_{i 4}-k b_{1 i}$. In particular,

$$
b_{12}^{\prime}=b_{12}, \quad b_{34}^{\prime}=b_{34}-k b_{13}, \quad b_{13}^{\prime}=b_{13}, \quad b_{24}^{\prime}=b_{24}-k b_{12} .
$$

Hence (ii) holds if $k \neq 0$, since $b_{13}^{\prime}, b_{24}^{\prime}$ are rationally independent. Further (i) will hold if we choose $k$ so that $-k b_{13}>0$.

The proof in the other cases is similar. In particular if $b_{13}=0$ but $b_{14} \neq 0$ we use a base change that alters $\lambda_{3}^{*}$ instead of $\lambda_{4}^{*}$.

Remark 3.7 Note that

$$
\omega \wedge \omega=\left(b_{13} b_{24}-b_{14} b_{23}-b_{12} b_{34}\right) \lambda_{1}^{*} \wedge \lambda_{3}^{*} \wedge \lambda_{2}^{*} \wedge \lambda_{4}^{*} .
$$

Since the base change was orientation-preserving, the coefficient is still positive, and so in the new basis for $\Lambda$ constructed in Lemma 3.6 we necessarily have $b_{13}^{\prime} b_{24}^{\prime}-b_{14}^{\prime} b_{23}^{\prime}>$ 0 , since $-b_{12}^{\prime} b_{34}^{\prime} \leq 0$ by (i). 
Proof of Proposition 3.2 We are given a torus $T^{4}=\mathbb{R}^{4} / \Lambda$ with a linear irrational symplectic form. We assume that we have chosen a basis $\lambda_{1}, \ldots, \lambda_{4}$ for $\Lambda$ such that the matrix $B$ determined from $\omega\left(\lambda_{i}, \lambda_{j}\right)=b_{i j}$ satisfies the conditions stated in Lemma 3.6. Our goal is to identify $\Lambda$ with a suitable period lattice $\Lambda_{P}$ of the form discussed in Lemma 3.5, where the coefficients $p, q, r, s$ are still to be determined. Moreover, we want that under this identification the form $\omega$ is as in (3-2) for suitable constants $x, y, u, v \in \mathbb{R}$, and is compatible with the standard complex structure $J_{0}$ on $\mathbb{C}^{2}$.

With respect to the real standard basis $e_{1}, e_{2}, e_{3}=\sqrt{-1} e_{1}, e_{4}=\sqrt{-1} e_{2}$ of $\mathbb{C}^{2}$ the symmetric bilinear form $g$ associated to a symplectic form $\omega$ as in (3-2) and the standard complex structure $J_{0}$ is represented by the matrix:

$$
\left(g_{i j}\right)=\omega\left(e_{i}, J_{0} e_{j}\right)=\left(\begin{array}{rrrr}
x & u & 0 & -v \\
u & y & v & 0 \\
0 & v & x & u \\
-v & 0 & u & y
\end{array}\right)
$$

The compatibility of $\omega$ with $J_{0}$ requires this matrix to be positive definite, and this holds if and only if all leading principal minors are positive. This will be the case if and only if

$$
x>0 \text { and } \quad x y-u^{2}-v^{2}>0,
$$

since the other two conditions $x y-u^{2}>0$ and $\left(x y-u^{2}-v^{2}\right)^{2}$ are then necessarily also satisfied.

To construct the lattice $\Lambda_{P}$ and find the coefficients of $\omega$, a comparison with equation (3-3) of the proof of Lemma 3.5 shows that we want to solve the equations

$$
\begin{aligned}
b_{12} & =v, & b_{13} & =p x+q u, \\
b_{14} & =r x+s u, & b_{23} & =p u+q y, \\
b_{24} & =r u+s y, & b_{34} & =v(p s-q r) .
\end{aligned}
$$

The middle four equations can be rewritten as:

$$
\left(\begin{array}{lll}
q & p & 0 \\
s & r & 0 \\
p & 0 & q \\
r & 0 & s
\end{array}\right)\left(\begin{array}{l}
u \\
x \\
y
\end{array}\right)=\left(\begin{array}{l}
b_{13} \\
b_{14} \\
b_{23} \\
b_{24}
\end{array}\right)
$$

Here the vector on the right hand side is given and nonzero. For fixed $p, q, r, s$ with $p s-q r \neq 0$ this overdetermined system of equations will have a solution $(u, x, y)$ if 
the compatibility condition

$$
r b_{13}-p b_{14}=q b_{24}-s b_{23}
$$

is satisfied.

Lemma 3.8 If the vector $\left(b_{13}, b_{14}, b_{23}, b_{24}\right)$ is not a multiple of a rational vector, there exists a solution $\left(p^{\prime}, q^{\prime}, r^{\prime}, s^{\prime}\right) \in \mathbb{R}^{4}$ of (3-6) in rationally independent real numbers satisfying $s^{\prime} b_{13}-q^{\prime} b_{14}>0$ and $D:=p^{\prime} s^{\prime}-q^{\prime} r^{\prime}>0$.

Proof The inequalities $s b_{13}-q b_{14}>0$ and $D>0$ define an open set in $\mathbb{R}^{4}$, which we denote by $\mathcal{O}$. Similarly, for given $b_{i j}$ the equation (3-6) defines a hyperplane $H$ in $\mathbb{R}^{4}$. The intersection $\mathcal{O} \cap H$ is not empty, since in view of Remark 3.7 the point with coordinates $p=b_{13}, q=b_{23}, r=b_{14}$ and $s=b_{24}$ belongs to it.

On the other hand, a point $(p, q, r, s)$ has rationally dependent coordinates if and only if it solves some equation $n_{1} p+n_{2} q+n_{3} r+n_{4} s=0$ with integral coefficients $n_{i}$. Since its defining vector is not a (multiple of a) rational vector, $H$ is transverse to this countable set of hyperplanes, and so there is some point in the open subset $\mathcal{O} \cap H$ that does not lie on any of these hyperplanes. This point has the desired properties.

Given $\left(p^{\prime}, q^{\prime}, r^{\prime}, s^{\prime}\right)$ as in the lemma, the solution of the matrix equation (3-5) is

$$
\begin{gathered}
x^{\prime}=\frac{1}{D}\left(s^{\prime} b_{13}-q^{\prime} b_{14}\right), \quad y^{\prime}=\frac{1}{D}\left(p^{\prime} b_{24}-r^{\prime} b_{23}\right), \\
u^{\prime}=\frac{1}{D}\left(p^{\prime} b_{14}-r^{\prime} b_{13}\right)=\frac{1}{D}\left(s^{\prime} b_{23}-q^{\prime} b_{24}\right) .
\end{gathered}
$$

If $b_{12}=b_{34}=0$, the final two equations

$$
b_{12}=v, \quad b_{34}=v\left(p^{\prime} s^{\prime}-q^{\prime} r^{\prime}\right),
$$

have the trivial solution $v=0$. In this case choose $\rho>0$ such that $\rho^{2}\left(p^{\prime} q^{\prime}-r^{\prime} s^{\prime}\right)$ is irrational, and define $(p, q, r, s):=\rho\left(p^{\prime}, q^{\prime}, r^{\prime}, s^{\prime}\right)$ and $(u, x, y):=\rho^{-1}\left(u^{\prime}, x^{\prime}, y^{\prime}\right)$.

If $b_{12}, b_{34}$ are rationally independent, we need to rescale the above solution so that $p s-q r=b_{34} / b_{12}$. Therefore, define $(p, q, r, s):=\rho\left(p^{\prime}, q^{\prime}, r^{\prime}, s^{\prime}\right)$ and $(u, x, y):=$ $\rho^{-1}\left(u^{\prime}, x^{\prime}, y^{\prime}\right)$, where $\rho:=\sqrt{b_{34} /\left(b_{12} D\right)}$. Notice that $p s-q r=b_{34} / b_{12}$ is automatically irrational by part (i) of Lemma 3.6 in this case. Now, choosing $v=b_{12}$, all six equations are satisfied.

With this construction, we have found a lattice $\Lambda_{P}$ and coefficients $x, y, u, v \in \mathbb{R}$ such that, after identifying $\Lambda$ with $\Lambda_{P}$ by mapping the basis vectors $\lambda_{j}$ of $\Lambda$ to the basis vectors $\lambda_{j}$ of $\Lambda_{P}$, the symplectic form $\omega$ is as in (3-2). To check the 
positivity condition (3-4), note that $x>0$ by construction, and a computation shows that $x y-u^{2}-v^{2}$ equals a positive multiple of $b_{13} b_{24}-b_{14} b_{23}-b_{12} b_{34}$, which was observed to be positive in Remark 3.7. In summary, we have shown that $\omega$ is compatible with the standard complex structure $J_{0}$ on $\mathbb{C}^{2}$. Finally, the lattice $\Lambda_{P}$ by construction satisfies the assumptions of Lemma 3.5, and so we have proven the proposition.

\subsection{Product tori}

In this section, we complete the proof of Theorem 1.9 by treating the case of product tori $T(1, \mu)$. We can assume that $\mu \geq 1$. If $\mu$ is irrational, Theorem 1.9 has been proven in Sections 3.1 and 3.2. If $\mu=1$ or $\mu \geq 2$ is rational, Theorem 1.9 follows from Proposition 3.9 below (notice that for $\mu \geq 2$ the condition on the individual $a_{j}$ and $b_{j}$ appearing in this proposition are automatic from volume considerations). Finally, if $\mu \in(1,2)$ is rational, Theorem 1.9 follows from the case of rational $\mu \geq 2$ as in the proof of Corollary 1.7.

Proposition 3.9 Let $\mu \geq 1$ be rational and let $\bar{B}\left(a_{1}\right), \ldots, \bar{B}\left(a_{k}\right)$ be a collection of balls such that

$$
\operatorname{Vol}\left(\coprod_{j=1}^{k} \bar{B}\left(a_{j}\right)\right)<\mu
$$

and such that $a_{j}<\mu$ for all $j$. Then there exists a symplectic embedding of $\coprod_{j=1}^{k} \bar{B}\left(a_{j}\right)$ into the open disc bundle $T^{2}(1) \times D^{2}(\mu)$.

Proof Denote by $S^{2}(\mu)$ the 2 -sphere endowed with an area form of area $\mu$. Biran has shown in [4, Proof of Corollary 5.C] that $\coprod_{j=1}^{k} \bar{B}\left(a_{j}\right)$ symplectically embeds into $T^{2}(1) \times S^{2}(\mu)$ whenever

$$
\operatorname{Vol}\left(\coprod_{j=1}^{k} \bar{B}\left(a_{j}\right)\right)<\mu
$$

and $a_{j}<\mu$ for all $j$. We will arrange this embedding in such a way that the balls lie in $T^{2}(1) \times\left(S^{2}(\mu) \backslash z_{0}\right)=T^{2}(1) \times D^{2}(\mu)$, where $z_{0} \in S^{2}$. Such a construction has been carried out in [5] in a slightly different situation. We shall outline the construction, pointing out the difference.

A symplectic embedding of $\coprod_{j=1}^{k} \bar{B}\left(a_{j}\right)$ into $T^{2}(1) \times\left(S^{2}(\mu) \backslash z_{0}\right)$ is obtained by constructing a smooth family of cohomologous forms $\omega_{s}, s \in[0,1]$, on $T^{2} \times S^{2}$ with the following properties:

- $\omega_{0}$ is the product form on $T^{2}(1) \times S^{2}(\mu)$, 
- each $\omega_{s}$ is nondegenerate on the torus $Z:=T^{2} \times\left\{z_{0}\right\}$,

- for each $s \in[0,1]$ there exists a symplectic embedding of $\coprod_{j=1}^{k} s \bar{B}\left(a_{j}\right)$ into $\left(\left(T^{2} \times S^{2}\right) \backslash Z, \omega_{S}\right)$.

For then a standard Moser argument shows that there is a family of diffeomorphisms $\psi_{s}:\left(T^{2} \times S^{2}, Z\right) \rightarrow\left(T^{2} \times S^{2}, Z\right)$ such that $\psi_{1}^{*} \omega_{1}=\omega_{0}$. Therefore $\coprod_{j=1}^{k} \bar{B}\left(a_{j}\right)$ symplectically embeds into $\left(T^{2} \times\left(S^{2} \backslash z_{0}\right), \omega_{0}\right)=T^{2}(1) \times D^{2}(\mu)$.

The family $\omega_{s}$ is constructed in much the same way as in the proof of Theorem 1.9 for irrational tori. In other words, the problem is converted into one of constructing suitable forms $\tau_{s}$ on the $k$-fold blow-up. The only difference is that we can no longer find the required forms $\tau_{s}$ on the blow-up via the Buchdahl-Lamari criterion; instead we must use symplectic inflation as in Lalonde and McDuff [24], McDuff [31], and Biran [4]. In order for $Z$ to be $\tau_{s}$-symplectic, it suffices to work only with almost complex structures $J$ for which $Z$ is $J$-holomorphic.

More precisely, let $\left(M, \omega_{0}\right):=T^{2}(1) \times S^{2}(\mu)$, and choose different points $x_{1}, \ldots, x_{k}$ in $M \backslash Z$. Let $J_{0}$ be the standard product complex structure on $M$, and choose neighborhoods $U(Z)=T^{2} \times D\left(z_{0}\right), B_{\varepsilon}\left(x_{1}\right), \ldots, B_{\varepsilon}\left(x_{k}\right)$ with disjoint closures. At each point $x_{j}$, form the Kähler blow-up of size $\varepsilon$. Denote the resulting Kähler manifold by $\left(M_{k}, J_{k}, \tau_{\varepsilon}\right)$. Let $\Sigma_{1}, \ldots, \Sigma_{k}$ be the exceptional divisors, and let $E_{j}$ be the homology class of $\Sigma_{j}$. Note that

$$
\pi: M_{k} \backslash \bigcup_{j} \Sigma_{j} \rightarrow M \backslash \bigcup_{j} x_{j}
$$

is a diffeomorphism, and denote $\pi^{-1}(Z)$ and $\pi^{-1}(U(Z))$ by $\tilde{Z}$ and $U(\tilde{Z})$. Choose mutually disjoint neighborhoods $U\left(\Sigma_{j}\right)$ that are also disjoint from $\tilde{Z}$. Let $\mathcal{J}$ be the space of $\tau_{\varepsilon}$-tame almost complex structures on $M_{k}$, and denote by $\mathcal{J}^{\prime}$ the subspace of those $J$ in $\mathcal{J}$ that restrict to $J_{k}$ on $U(\widetilde{Z}) \cup U\left(\Sigma_{1}\right) \cup \cdots \cup U\left(\Sigma_{k}\right)$. As in [4; 5], the class $\left[\omega_{0}\right] \in H^{2}\left(T^{2} \times S^{2} ; \mathbb{R}\right)$ is rational by assumption. Choose rational numbers $a_{j}^{\prime} \in\left(a_{j}, \mu\right)$ such that $\operatorname{Vol}\left(\coprod_{j=1}^{k} \bar{B}\left(a_{j}\right)\right)<\mu$. We then find $n \in \mathbb{N}$ such that

$$
A:=n\left(\mathrm{PD}\left[\pi^{*} \omega_{0}\right]-\sum_{j=1}^{k} a_{j}^{\prime} E_{j}\right)
$$

belongs to $H_{2}\left(M_{k} ; \mathbb{Z}\right)$. In order to construct the forms $\tau_{s}$ we wish to inflate the form $\tau_{\varepsilon}$ along the class $A$. To this end we need to represent $A$ by a smooth connected embedded reduced $J$-holomorphic curve $C$ for some $J \in \mathcal{J}^{\prime}$. To find such a curve $C$ one can closely follow the proof of [5, Lemma 2.2.B], where, however, the curve $\tilde{Z}$ has negative self-intersection. This causes no problem if one observes that, by the maximum principle, for every $J^{\prime} \in \mathcal{J}^{\prime}$, every $J^{\prime}$-holomorphic curve that is entirely contained 
in $U(\tilde{Z})$ must be (a multiple cover of) a torus $\pi^{-1}\left(T^{2} \times\{z\}\right)$ with $z \in D\left(z_{0}\right)$. For $B \in H_{2}\left(M_{k} ; \mathbb{Z}\right)$, the maximal number of generic points through which, for generic $J \in \mathcal{J}$, a $J$-holomorphic curve can pass, is $k(B):=\frac{1}{2}\left(B \cdot B+c_{1}(B)\right)$. Let

$$
A=\sum_{j} m_{j} A_{j}+m[\tilde{Z}], \quad m \geq 1,
$$

be an $A$-cusp configuration with $m \geq 1$. Denote by $k_{\text {cusp }}(A)$ the maximal number of generic points through which, for a generic subset of $J^{\prime}$ in $\mathcal{J}^{\prime}$, a $J^{\prime}$-cusp-curve with configuration (3-8) can pass. As in [5, pp. 148-151], the last step in the existence proof for the curve $C$ is to understand that $k_{\text {cusp }}(A)<k(A)$. To see this, consider the class $\bar{A}:=A-m[\tilde{Z}]$. In view of (3-7), the class $A$ is not a multiple of $[\tilde{Z}]$, and hence the class $\bar{A}$ is non-trivial. Biran showed in the proof of Lemma 2.2.B of [5] that for generic $J^{\prime} \in \mathcal{J}^{\prime}$, no (cusp-)curve in class $\bar{A}$ can pass through more than $k(\bar{A})$ generic points. Since in the definition of $k_{\text {cusp }}(A)$ we may consider points outside $U(\tilde{Z})$ only, it follows that $k_{\text {cusp }}(A) \leq k(\bar{A})$. Moreover, using $c_{1}(\tilde{Z})=0,[\tilde{Z}] \cdot[\tilde{Z}]=0$ and $A \cdot[\widetilde{Z}]=n \int_{Z} \omega_{0}>0, m \geq 1$, we compute

$$
k(\bar{A})=k(A)-m \bar{A} \cdot[\tilde{Z}]=k(A)-m A \cdot[\tilde{Z}]<k(A) .
$$

Altogether, $k_{\text {cusp }}(A) \leq k(\bar{A})<k(A)$.

\section{Basic symplectic mappings}

In this section we describe an elementary symplectic embedding construction. It will be applied in Section 5 to prove that $p(T(1,1))=1$. We write $\mathbb{R}^{2}(\boldsymbol{x}):=\mathbb{R}^{2}\left(x_{1}, x_{2}\right)$ and $\mathbb{R}^{2}(\boldsymbol{y}):=\mathbb{R}^{2}\left(y_{1}, y_{2}\right)$.

\subsection{Diamonds}

Consider the "diamond" of size $a$

$$
\diamond(a):=\left\{\left(x_{1}, x_{2}\right) \in \mathbb{R}^{2}(\boldsymbol{x})|| x_{1}|+| x_{2} \mid<\frac{a}{2}\right\} \subset \mathbb{R}^{2}(\boldsymbol{x}) ;
$$

see Figure 3(I).

Lemma 4.1 For each $\varepsilon>0$ the ball $B^{4}(a)$ symplectically embeds into

$$
\diamond(a+\varepsilon) \times(0,1)^{2} \subset \mathbb{R}^{2}(\boldsymbol{x}) \times \mathbb{R}^{2}(\boldsymbol{y}) .
$$


Proof Let $D(a) \subset \mathbb{R}^{2}(z)=\mathbb{R}^{2}(x, y)$ be the open disc of area $a$. Choose an area and orientation-preserving embedding

$$
\sigma: D(a) \rightarrow\left(-\frac{a+\varepsilon}{2}, \frac{a+\varepsilon}{2}\right) \times(0,1)
$$

such that

$$
|x(\sigma(z))|<\frac{1}{2} \pi|z|^{2}+\frac{\varepsilon}{2} \quad \text { for all } z \in D(a) .
$$

Figure 1 shows such an embedding. For details we refer to [36, Lemma 3.1.8].
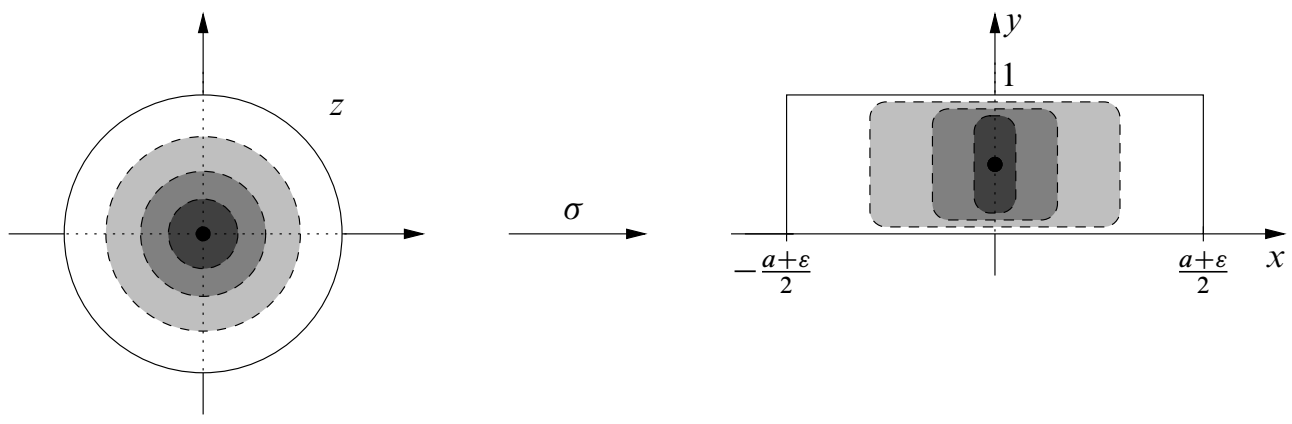

Figure 1: The map $\sigma$

We claim that the symplectic embedding $\sigma \times \sigma: D(a) \times D(a) \rightarrow \mathbb{R}^{4}$ maps $B^{4}(a)$ to $\diamond(a+\varepsilon) \times(0,1)^{2}$. Indeed, for $\left(z_{1}, z_{2}\right) \in B^{4}(a)$ we have $\pi\left(\left|z_{1}\right|^{2}+\left|z_{2}\right|^{2}\right)<a$. Together with (4-1) we can estimate

$$
\begin{aligned}
\left|x_{1}\left((\sigma \times \sigma)\left(z_{1}, z_{2}\right)\right)\right|+\left|x_{2}\left((\sigma \times \sigma)\left(z_{1}, z_{2}\right)\right)\right| & =\left|x_{1}\left(\sigma\left(z_{1}\right)\right)\right|+\left|x_{2}\left(\sigma\left(z_{2}\right)\right)\right| \\
& <\frac{1}{2}\left(\pi\left|z_{1}\right|^{2}+\pi\left|z_{2}\right|^{2}\right)+\varepsilon \\
& <\frac{a}{2}+\varepsilon
\end{aligned}
$$

as claimed.

Corollary 4.2 The open ball $B^{4}(a)$ is symplectomorphic to

$$
\diamond(a) \times(0,1)^{2} \subset \mathbb{R}^{2}(\boldsymbol{x}) \times \mathbb{R}^{2}(\boldsymbol{y}) .
$$

Proof This follows by combining the above lemma with Lemma 4.3 below.

Lemma 4.3 Let $V \subset \mathbb{R}^{4}$ be a bounded domain such that for each compact subset $K \subset V$ there exists $\hat{a}<a$ and a symplectic embedding $\hat{\varphi}: B^{4}(\hat{a}) \rightarrow V$ such that $\operatorname{Im} \hat{\varphi} \supset K$. Then $V$ is symplectomorphic to $B^{4}(a)$. 
Proof Choose a sequence $K_{1} \subset K_{2} \subset \cdots$ of compact subsets of $V$ such that $\bigcup_{j} K_{j}=V$. Using the assumption of the lemma and the result from [30] that the space of symplectic embeddings of a closed ball into an open ball is connected, we construct a sequence $a_{1}^{\prime}<a_{1}<a_{2}^{\prime}<a_{2}<\cdots$ with $a_{j} \rightarrow a$ and a sequence of symplectic embeddings $\varphi_{j}: B^{4}\left(a_{j}\right) \rightarrow V$ such that $\varphi_{j}\left(B^{4}\left(a_{j}^{\prime}\right)\right) \supset K_{j}$ and $\left.\varphi_{j+1}\right|_{B^{4}\left(a_{j}^{\prime}\right)}=\left.\varphi_{j}\right|_{B^{4}\left(a_{j}^{\prime}\right)}$.

Define $\varphi: B^{4}(a) \rightarrow V$ by $\varphi(z)=\varphi_{j}(z)$ if $z \in B^{4}\left(a_{j}^{\prime}\right)$. Then $\varphi$ is a well-defined symplectic embedding. Also, $\varphi\left(B^{4}\left(a_{j}^{\prime}\right)\right)=\varphi_{j}\left(B^{4}\left(a_{j}^{\prime}\right)\right) \supset K_{j}$. Hence $\varphi$ is onto $\bigcup_{j} K_{j}=V$.

\subsection{Distorted diamonds}

All of our embeddings, besides one, will start from a diamond $\diamond(a)$. For our full filling of $T(1,1)$, however, we shall need to start from a distorted diamond.

Fix $a>0$. Let $u_{+}:[0, a] \rightarrow \mathbb{R}$ be a continuous, nondecreasing function that for convenience we take to be piecewise-linear. Suppose further that

$$
u_{+}(0)=0 \quad \text { and } \quad u_{+}^{\prime}(\rho) \in[0,1] \text { on the linear pieces. }
$$

Define the piecewise-linear function $u_{-}:[0, a] \rightarrow \mathbb{R}$ by $u_{-}(\rho)=u_{+}(\rho)-\rho$. Then $u_{-}(0)=0$ and $u_{-}^{\prime}(\rho) \in[-1,0]$, so that $u_{-}$is nonincreasing. Moreover, $u_{+}^{\prime}(\rho)-$ $u_{-}^{\prime}(\rho)=1$ on the linear pieces, and $u_{+}(a)-u_{-}(a)=a$. Let

$$
\sigma_{u}: D(a) \rightarrow\left(u_{-}(a)-\frac{\varepsilon}{2}, u_{+}(a)+\frac{\varepsilon}{2}\right) \times(0,1)
$$

be a symplectic embedding such that

$$
x\left(\sigma_{u}(z)\right) \in\left(u_{-}(\rho)-\frac{\varepsilon}{2}, u_{+}(\rho)+\frac{\varepsilon}{2}\right) \text { for all } z \in D(a) \text { with } \pi|z|^{2}<\rho .
$$

Figure 2 shows the image of concentric circles of the map $\sigma_{u}$ for $a=3$ and for the function $u_{+}:[0,3] \rightarrow \mathbb{R}$ with slope $\frac{1}{2}$ on $\left[0, \frac{3}{2}\right]$ and slope $\frac{1}{3}$ on $\left[\frac{3}{2}, 3\right]$.

Let $u_{+}$and $v_{+}$be functions as above, and

$$
\sigma_{u}: D(a) \rightarrow \mathbb{R}^{2}\left(x_{1}, y_{1}\right), \quad \sigma_{v}: D(a) \rightarrow \mathbb{R}^{2}\left(x_{2}, y_{2}\right)
$$

be symplectic embeddings associated to $u$ and $v$ as above. Then, as in Lemma 4.1, the product $\sigma_{u} \times \sigma_{v}$ induces a symplectic embedding $B^{4}(a) \rightarrow \mathbb{R}^{2}(\boldsymbol{x}) \times(0,1)^{2}$. Because $u_{+}, v_{+}$are piecewise linear, the image $\left(\sigma_{u} \times \sigma_{v}\right)\left(B^{4}(a)\right)$ of the ball projects to a ( $\varepsilon$-neighbourhood of a) polygon in $\mathbb{R}^{2}(\boldsymbol{x})$ whose vertices are determined by the nonsmooth points of $u$ and $v$. For example, if $u_{+}(\rho)=v_{+}(\rho)=\frac{\rho}{2}$ then the image is the standard diamond $\diamond(a)$ constructed above. 


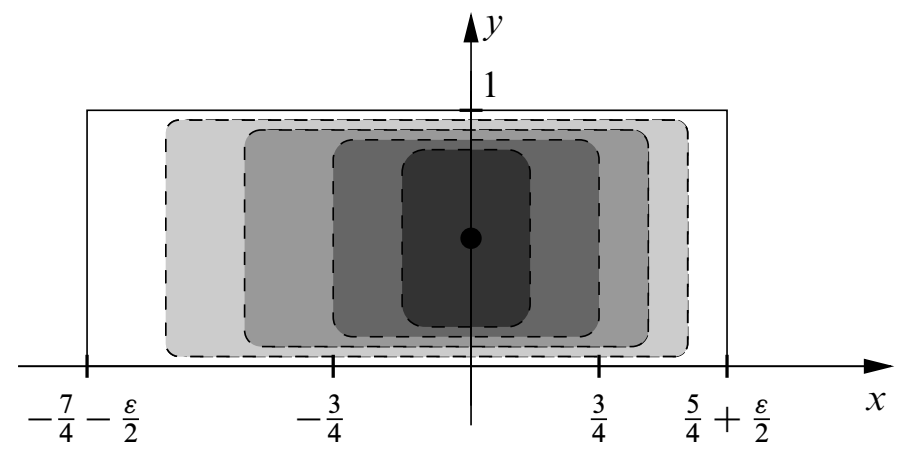

Figure 2: The map $\sigma_{u}$ for a function $u_{+}$with two pieces

Assume now that $a>1$. Define $d$ by $2 d=a-1$, and suppose that the functions $u_{+}, v_{+}$have only two pieces, where $u_{+}$is standard (ie, equal to $\frac{\rho}{2}$ ) on $[0,2 d]$ and $v_{+}$is standard on $[0,1]$. Then the distortion occurs when either $\left|x_{1}\right|>d$ or $\left|x_{2}\right|>\frac{1}{2}$. Computing as in the proof of Lemma 4.1 (again omitting $\varepsilon>0$ ), we find that the image of $B^{4}(a)$ under $\sigma_{u} \times \sigma_{v}$ is contained in $\diamond \times(0,1)^{2}$, where $\diamond$ is as in Figure 3(II).

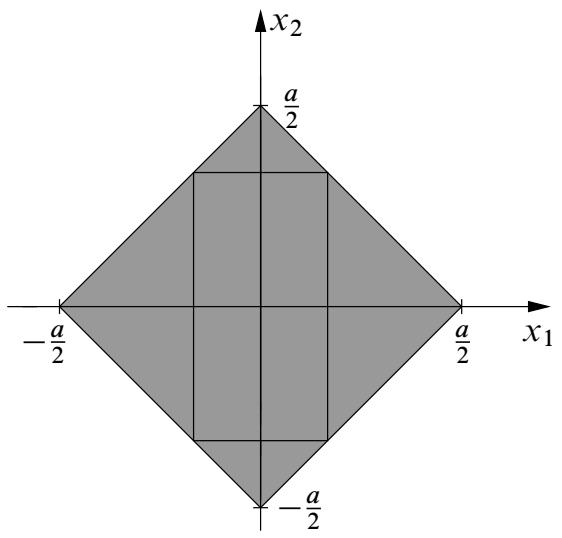

(I)

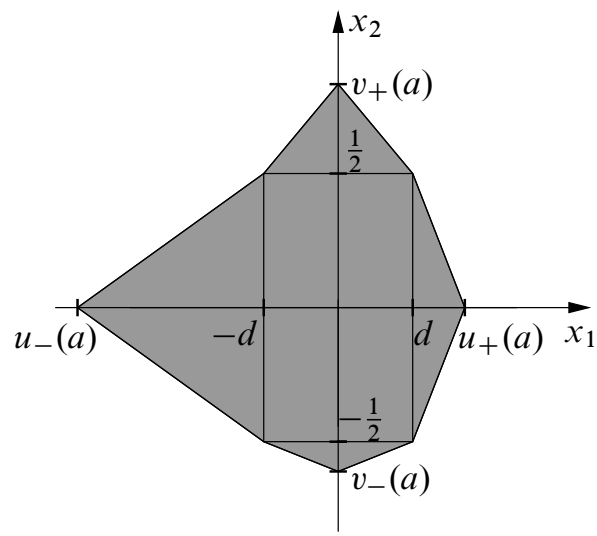

(II)

Figure 3: The diamond $\diamond(a)$ and a distorted diamond

We call the factor $\diamond$ of such an image $\diamond \times(0,1)^{2}$ a distorted diamond. A distorted diamond of size $a>1$ therefore consists of:

- A rectangle $(-d, d) \times\left(-\frac{1}{2}, \frac{1}{2}\right)$ with $2 d=a-1$.

- A top and bottom triangle each with base $2 d$, the sum of whose heights is $v_{+}(a)-v_{-}(a)-1=a-1=2 d$. 
- Two flaps each with height 1 , the sum of whose widths is $u_{+}(a)-u_{-}(a)-2 d=$ $a-(a-1)=1$.

Corollary 4.2 has the following generalization.

Proposition 4.4 Let $\diamond$ be a distorted diamond of size $a$. Then the product $\diamond \times(0,1)^{2}$ is symplectomorphic to the open ball $B^{4}(a)$.

Proof In view of the above construction, for each compact subset $K \subset \diamond \times(0,1)^{2}$ there exists $\hat{a}<a$ and a symplectic embedding $\hat{\varphi}: B^{4}(\hat{a}) \rightarrow \diamond \times(0,1)^{2}$ such that $\operatorname{Im} \hat{\varphi} \supset K$. The proposition therefore again follows from Lemma 4.3.

In [40], Traynor used a different construction to prove Proposition 4.4 for the special case that $\diamond$ is the standard simplex $\left\{\left(x_{1}, x_{2}\right) \mid x_{1}, x_{2}>0, x_{1}+x_{2}<a\right\}$.

\subsection{Shears}

Let $f: \mathbb{R} \rightarrow \mathbb{R}$ be a smooth function. Consider the $x_{1}$-shear

$$
\varphi\left(x_{1}, x_{2}\right)=\left(x_{1}+f\left(x_{2}\right), x_{2}\right)
$$

of $\mathbb{R}^{2}$. Then the diffeomorphism

$$
\widehat{\varphi}\left(x_{1}, x_{2}, y_{1}, y_{2}\right)=\left(x_{1}+f\left(x_{2}\right), x_{2}, y_{1}, y_{2}-f^{\prime}\left(x_{2}\right) y_{1}\right)
$$

is a symplectomorphism of $\mathbb{R}^{4}$. Indeed, this is just the "cotangent map"

$$
\left(x_{1}, x_{2}, y_{1}, y_{2}\right) \mapsto\left(\varphi\left(x_{1}, x_{2}\right),\left(d \varphi\left(x_{1}, x_{2}\right)^{T}\right)^{-1}\left(y_{1}, y_{2}\right)\right)
$$

of the shear $\varphi$. We call a map $\hat{\varphi}$ of the form (4-2) also an $x_{1}$-shear. Similarly, an $x_{2}$-shear $\varphi\left(x_{1}, x_{2}\right)=\left(x_{1}, x_{2}+g\left(x_{1}\right)\right)$ induces a symplectomorphism

$$
\left(x_{1}, x_{2}, y_{1}, y_{2}\right) \mapsto\left(x_{1}, x_{2}+g\left(x_{1}\right), y_{1}-g^{\prime}\left(x_{1}\right) y_{2}, y_{2}\right),
$$

which we again call an $x_{2}$-shear.

Let $U \subset \mathbb{R}^{2}(\boldsymbol{x})$ be a domain, and consider the image $\tilde{\varphi}\left(U \times(0,1)^{2}\right)$ of an $x_{1}$-shear in $\mathbb{R}^{2}(\boldsymbol{x}) \times \mathbb{R}^{2}(\boldsymbol{y})$. This image fibers over $\varphi(U) \subset \mathbb{R}^{2}(\boldsymbol{x})$, with fiber $\left\{\left(y_{1}, y_{2}-\right.\right.$ $\left.\left.f^{\prime}\left(x_{2}\right) y_{1}\right) \mid\left(y_{1}, y_{2}\right) \in(0,1)^{2}\right\}$ over $\varphi\left(x_{1}, x_{2}\right)$; see Figure 4 .

The projection $\pi_{\mathbf{y}}: \mathbb{R}^{2}(\boldsymbol{y}) \rightarrow \mathbb{R}^{2}(\boldsymbol{y}) / \mathbb{Z}^{2}(1,1)$ is injective on these fibers. Further

$$
T(\mu, 1)=\mathbb{R}^{2}(\boldsymbol{x}) / \mathbb{Z}^{2}(\mu, 1) \times \mathbb{R}^{2}(\boldsymbol{y}) / \mathbb{Z}^{2}(1,1) .
$$




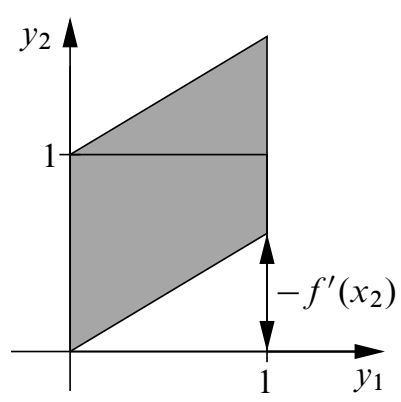

Figure 4: The fiber over $\varphi\left(x_{1}, x_{2}\right)$

It follows that if the projection $\pi_{x}: \mathbb{R}^{2}(x) \rightarrow \mathbb{R}^{2}(\boldsymbol{x}) / \mathbb{Z}^{2}(\mu, 1)$ is injective on $\varphi(U)$, then also

$$
\pi=\pi_{\boldsymbol{x}} \times \pi_{\boldsymbol{y}}: \tilde{\varphi}\left(U \times(0,1)^{2}\right) \rightarrow T(\mu, 1)
$$

is injective. The same holds true for $x_{2}$-shears.

One can check by direct calculation that an arbitrary composite of shears can map $U \times(0,1)^{2}$ to a set that intersects the fibers $\boldsymbol{x} \times \mathbb{R}^{2}(\boldsymbol{y})$ in subsets that no longer project injectively under $\pi_{\boldsymbol{y}}$. The next lemma gives conditions under which shears may be composed: One essential condition is that the shears must affect disjoint subsets of $U$.

Lemma 4.5 Suppose that an $x_{1}$-shear $\varphi_{1}$ and an $x_{2}$-shear $\varphi_{2}$ satisfy the following conditions:

(i) $\varphi_{2}$ fixes the set $\varphi_{1}\left(\left\{\boldsymbol{x} \in U \mid \varphi_{1}(\boldsymbol{x}) \neq \boldsymbol{x}\right\}\right)$ pointwise.

(ii) $\pi_{\boldsymbol{x}}$ injects $\varphi_{2} \circ \varphi_{1}(U)$ into $\mathbb{R}^{2}(\boldsymbol{x}) / \mathbb{Z}^{2}(\mu, 1)$.

Then $\pi$ injects $\hat{\varphi}_{2} \circ \widehat{\varphi}_{1}\left(U \times(0,1)^{2}\right)$ into $T(\mu, 1)$.

Proof The first condition implies that each 2-plane $\boldsymbol{x} \times \mathbb{R}^{2}(\boldsymbol{y})$ is moved by at most one of the shears. Hence each $\boldsymbol{x}$-fiber of the image $\hat{\varphi}_{2} \circ \widehat{\varphi}_{1}\left(U \times(0,1)^{2}\right)$ projects injectively under $\pi_{\boldsymbol{y}}: \mathbb{R}^{2}(\boldsymbol{y}) \rightarrow \mathbb{R}^{2}(\boldsymbol{y}) / \mathbb{Z}(1,1)$. It remains to check that the projection to $\mathbb{R}^{2}(\boldsymbol{x}) / \mathbb{Z}(\mu, 1)$ is injective, which is guaranteed by (ii).

We next give three examples illustrating the above embedding method.

Example 4.6 A full filling of $\boldsymbol{T}\left(\mathbf{2} \boldsymbol{k}^{\mathbf{2}}, \mathbf{1}\right)$ for each $\boldsymbol{k} \in \mathbb{N}$ We start from the diamond $\diamond(2 k)$, with vertices $( \pm k, 0),(0, \pm k)$. Using the linear shear $\varphi\left(x_{1}, x_{2}\right)=\left(x_{1}+\right.$ $\left.(2 k-1) x_{2}, x_{2}\right)$, the diamond is transformed into the parallelogram $P(k)$ with vertices $( \pm k, 0)$ and $\pm\left(2 k^{2}-k, k\right)$; see Figure 5 . 


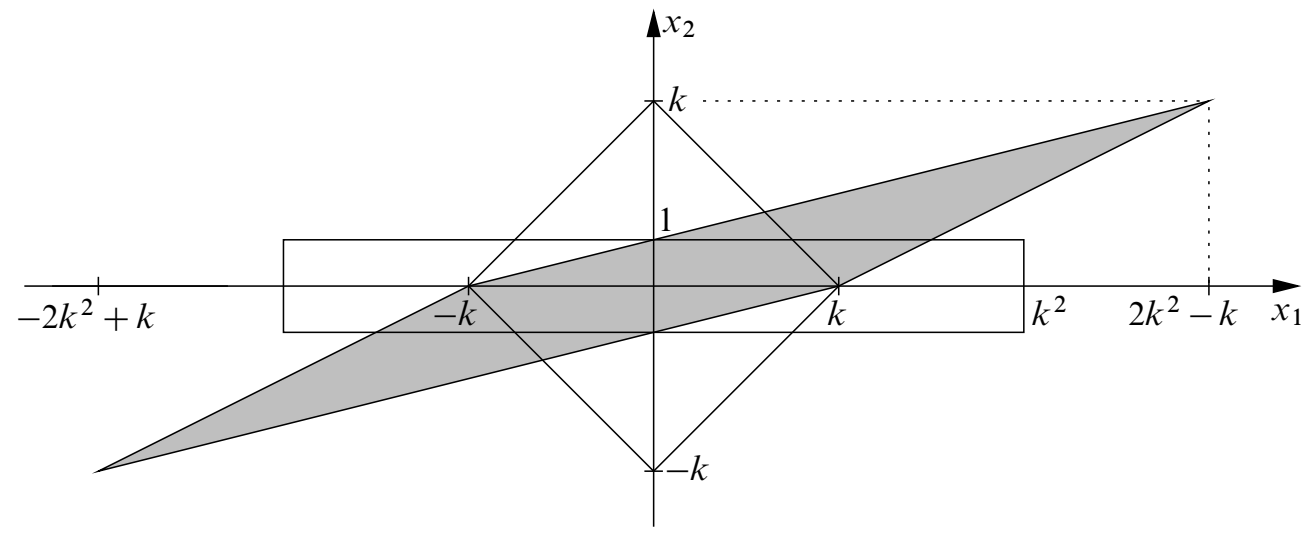

Figure 5: The parallelogram $P(k)$

This shear is chosen so that:

- the vertical distance between the top and bottom edges of $P(k)$ is 1 , and - each of these edges projects to an interval of length $2 k^{2}$ on the $x_{1}$-axis.

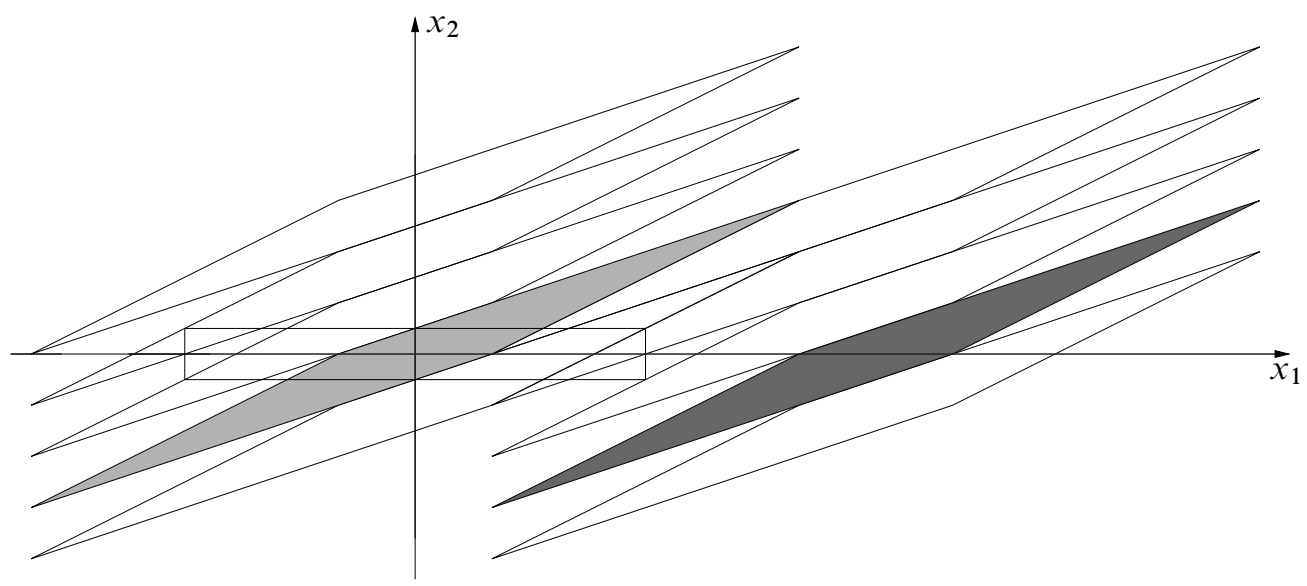

Figure 6: The tiling of $\mathbb{R}^{2}(\mathbf{x})$ by translates of $P(k)$ (in the picture $k=3$ ). The darker parallelogram is a translate of the lighter one by $2 k^{2}$ in the $x_{1}-$ direction. The rectangle marks the standard fundamental domain already depicted in Figure 5.

It follows that the set $P(k)$ is a fundamental domain for the action of $\mathbb{Z}^{2}$ with generators $2 k^{2} \partial_{x_{1}}$ and $\partial_{x_{2}}$; cf Figure 6. Now Proposition 4.4 shows that the ball $B^{4}(2 k)$ symplectically embeds into $T\left(2 k^{2}, 1\right)$. 
Remark 4.7 Together with scaling and Remark 2.2, this gives an explicit full filling by one ball of $T(\mu, 1)$ for all $\mu=2 m^{2} / n^{2}$ with $m, n$ relatively prime. (Non-explicit full fillings of these tori follow from the computation of Seshadri constants (2-8), and from Proposition 1.6, together with Remark 2.2.)

Remark 4.8 By shears as in Example 4.6 one can also construct explicit full fillings for some special irrational tori, namely for those of the form $\mathbb{R}^{4} / \Lambda$, where the lattice splits as $\Lambda=\Lambda_{x} \times \Lambda_{y}$, such that $\Lambda_{y}$ is the standard $\mathbb{Z}^{2} \subset \mathbb{R}^{2}(\boldsymbol{y})$ and such that some linearly sheared diamond in $\mathbb{R}^{2}(\boldsymbol{x})$ is a fundamental domain for $\Lambda_{x}$.

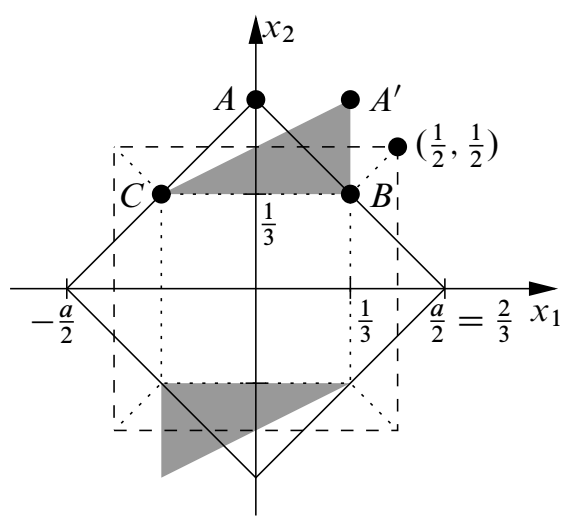

(I)

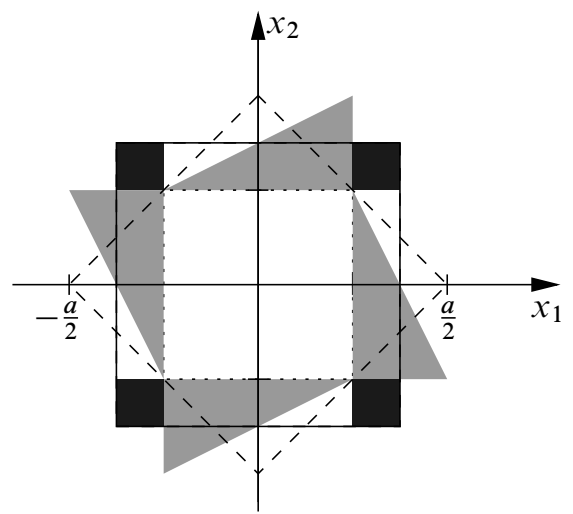

(II)

Figure 7: Filling $\frac{8}{9}$ of $T(1,1)$

Example 4.9 Filling $\frac{8}{9}$ of $\boldsymbol{T}(\mathbf{1}, \mathbf{1})$ Let $a=\frac{4}{3}$. The corners of the maximal inscribed square in $\diamond(a)$ with sides parallel to the axes have coordinates $\left( \pm \frac{1}{3}, \pm \frac{1}{3}\right)$. Two of these corners are labelled $B$ and $C$ in Figure 7(I). Choose $f$ with $f\left(x_{2}\right)=0$ for $-\frac{1}{3} \leq x_{2} \leq \frac{1}{3}$ and $f^{\prime}\left(x_{2}\right)=1$ for $\left|x_{2}\right|>\frac{1}{3}$ (with rounding between). In particular, $f\left(-\frac{a}{2}\right)=-\frac{1}{3}$ and $f\left(\frac{a}{2}\right)=\frac{1}{3}$. The $x_{1}$-shear induced by $f$ then takes the upper triangle $A B C$ to the shaded triangle $A^{\prime} B C$, and similarly for the bottom triangle, while the rest of the diamond is untouched. The $x_{2}$-shear induced by the function $-f$ then moves the left and right flaps of $\diamond(a)$ to the flaps shown in Figure 7(II), while the rest of the image of the $x_{1}$-shear is untouched. Therefore, a point in $\diamond(a)$ is affected by at most one of these two shears, so that we can apply Lemma 4.5.

The composition of these two shears takes (a slight shrinking of) $\diamond(a)$ to the shaded domain in Figure 7 (II). This domain injects into $\mathbb{R}^{2}(\boldsymbol{x}) / \mathbb{Z}^{2}(1,1)$ : It wraps up under the action of $\mathbb{Z}^{2}$ to a set covering all of the square $\left(-\frac{1}{2}, \frac{1}{2}\right)^{2}$ except the four black 
squares of area $\left(\frac{1}{6}\right)^{2}$ each. For each $\varepsilon>0$ we thus have constructed a symplectic embedding of a ball into $T(1,1)$ filling at least $\frac{8}{9}-\varepsilon$ of the volume of $T(1,1)$.

Example 4.10 Filling $\frac{49}{50}$ of $\boldsymbol{T}(\mathbf{1}, \mathbf{1})$ Let $a=\frac{7}{5}$. The idea is to divide the square representing $T(1,1)$ into two rectangles, one the maximum rectangle of height 1 that lies in the diamond $\diamond(a)$ (and hence has width $a-1=\frac{2}{5}$ ), and the other of width $2-a=\frac{3}{5}$; see Figure 8(I).

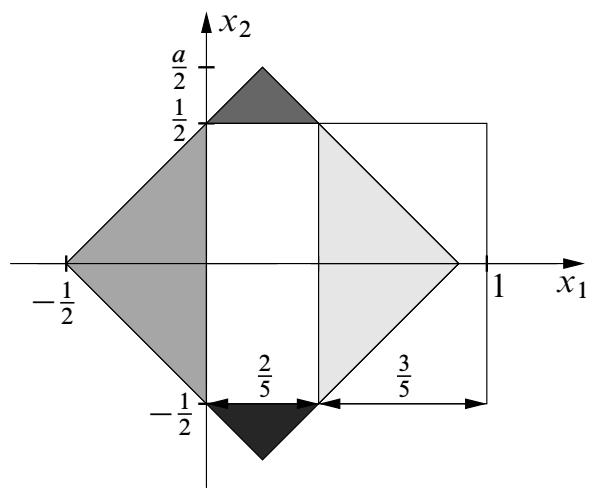

(I)

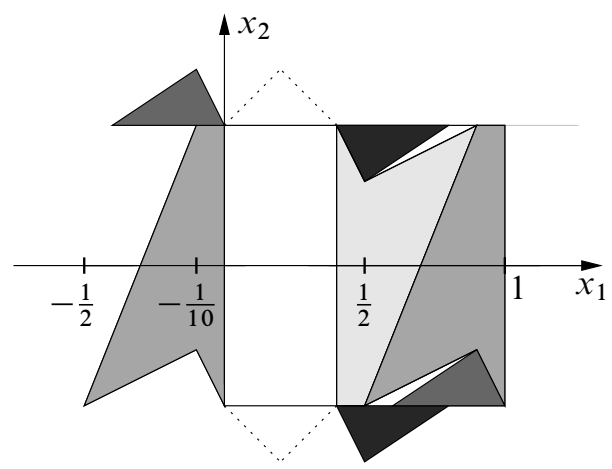

(II)

Figure 8: Filling $\frac{49}{50}$ of $T(1,1)$, schematically

We shear the top triangle by a strong $x_{1}$-shear to the left, the bottom triangle by a strong $x_{1}$-shear to the right, and then shear the flaps by $x_{2}$-shears in a symmetric way so as to free triangles into which the sheared top and bottom triangles fit, when projected to the torus. The freed triangles have height $\frac{1}{5}$ and width $\frac{1}{2}$, while the triangles fitting in have the same height, but width $\frac{2}{5}$ only. In Figure 8(II), one sees the image of the dark grey top triangle and its translate by $\partial_{x_{1}}-\partial_{x_{2}}$, the image of the black bottom triangle and its translate by $\partial_{x_{2}}$, as well as the image of the mid-grey left flap and its translate by $\partial_{x_{1}}$.

To make this construction precise, fix a small $\varepsilon>0$, and decompose the diamond $\diamond(a-\varepsilon)$ into four triangles and a rectangle of height 1 and width $\frac{2}{5}$ from each of whose four vertices a simplex of width $\frac{\varepsilon}{2}$ has been removed. For notational convenience, we also translate $\diamond(a-\varepsilon)$ by $\frac{1}{2} \partial_{x_{2}}$ (see Figure 9 ).

The $x_{1}$-shear, that moves the top triangle to the left and the bottom triangle to the right, has the following properties:

- It has support in $\left\{x_{2}>1-\frac{\varepsilon}{4}\right\} \cup\left\{x_{2}<0\right\}$. 


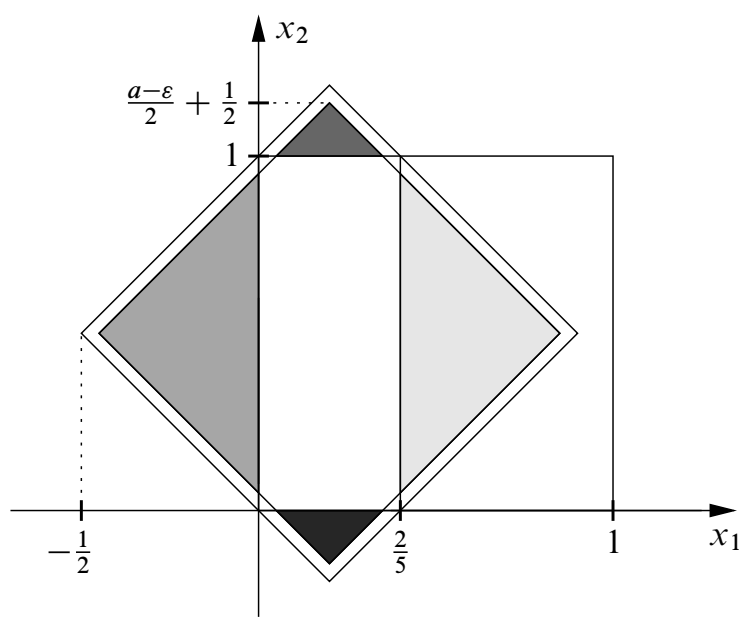

Figure 9: The decomposition of the diamond $\diamond(a-\varepsilon)$

- Near the upper triangle, this shear is very strong on $\left\{1-\frac{\varepsilon}{4}<x_{2}<1\right\}$, and up to a minor shear, it is a translation on $\left\{1<x_{2}<\frac{a-\varepsilon}{2}+\frac{1}{2}\right\}$.

- It fixes the points $A=\left(\frac{\varepsilon}{4}, 1-\frac{\varepsilon}{4}\right)$ and $B=\left(\frac{2}{5}-\frac{\varepsilon}{4}, 1-\frac{\varepsilon}{4}\right)$, but translates $C=\left(\frac{\varepsilon}{2}, 1\right)$ to $C^{\prime}=\left(-\frac{2}{5}+\frac{\varepsilon}{2}, 1\right)$ and $D=\left(\frac{2}{5}-\frac{\varepsilon}{2}, 1\right)$ to $D^{\prime}=\left(-\frac{\varepsilon}{2}, 1\right)$.

- On the bottom triangle, it acts very strongly on $\left\{-\frac{\varepsilon}{4}<x_{2}<0\right\}$.

- It fixes the points $X=\left(\frac{\varepsilon}{2}, 0\right)$ and $Y=\left(\frac{2}{5}-\frac{\varepsilon}{2}, 0\right)$, but translates $W=\left(\frac{3}{4} \varepsilon,-\frac{\varepsilon}{4}\right)$ to $W^{\prime}=\left(\frac{2}{5}+\frac{\varepsilon}{2},-\frac{\varepsilon}{4}\right)$ and $Z=\left(\frac{2}{5}-\frac{3}{4} \varepsilon,-\frac{\varepsilon}{4}\right)$ to $Z^{\prime}=\left(\frac{4}{5}-\varepsilon,-\frac{\varepsilon}{4}\right)$.

In Figure 10 we drew the projection of the (dark grey) top triangle $\left\{1-\frac{\varepsilon}{4}<x_{2}\right\}$ to the fundamental domain $(0,1) \times(0,1)$ of the usual $\mathbb{Z}^{2}$-action, but we did not draw the projection of the bottom triangle $\left\{x_{2}<0\right\}$. In order to see that the image projects injectively to $T(1,1)$, notice that:

- the point $C$ lies strictly above the segment $\overline{D^{\prime} B}$,

- $W^{\prime}$ lies on the right of $B$, and

- $B$ lies $\frac{\varepsilon}{4}$ below $C$ and $W^{\prime}$ lies $\frac{\varepsilon}{4}$ below $X$.

Therefore, the translate by $\partial_{x_{2}}$ of the segment $\overline{X W^{\prime}}$ lies above the segment $\overline{D^{\prime} B}$.

\section{Proof of Theorem 1.1}

Since we already proved Theorem 1.9 , it only remains to treat the product torus $T(1,1)$. So let $a=\sqrt{2}$. We want to find, for each $\varepsilon>0$, a symplectic embedding of the ball 


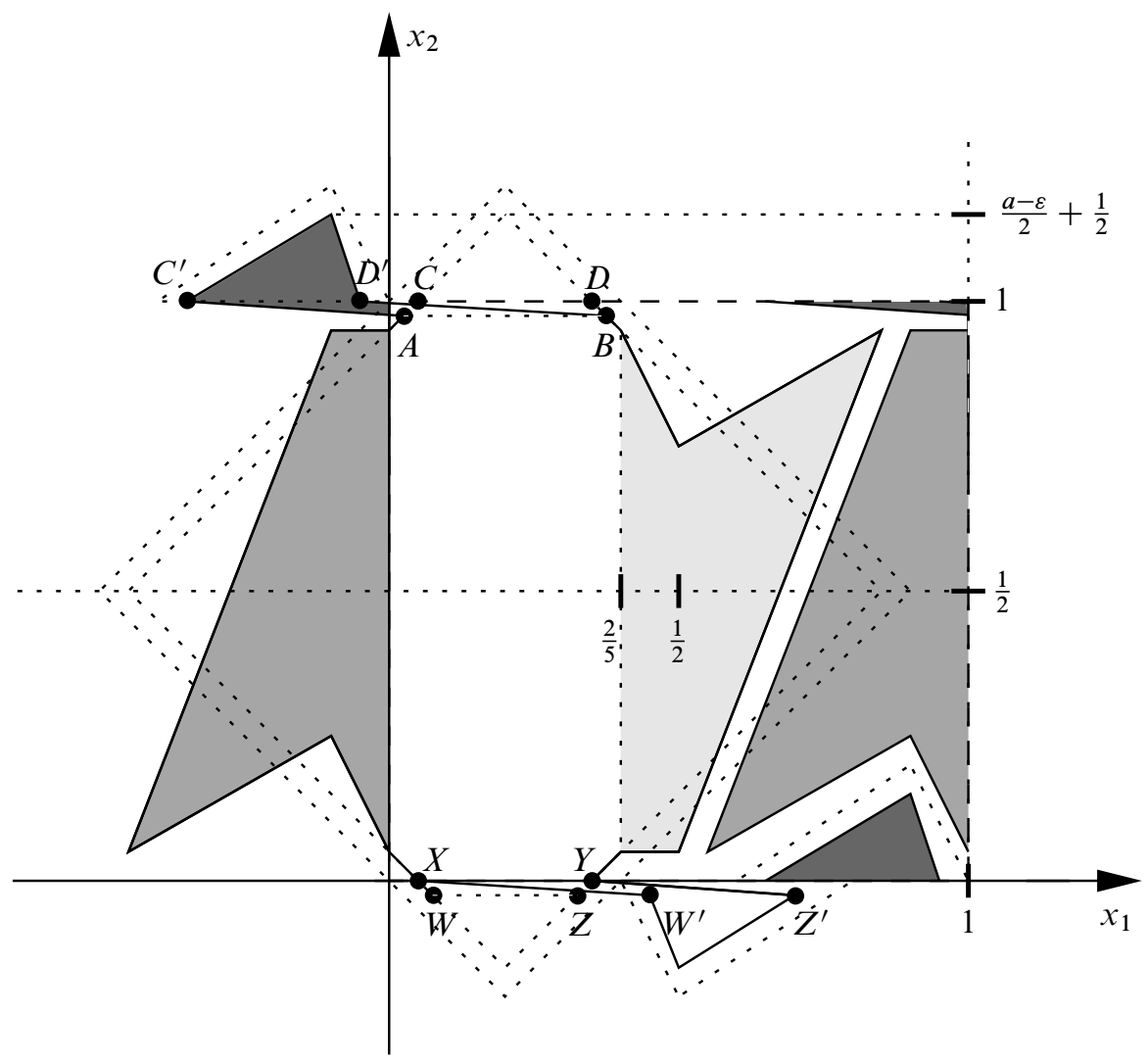

Figure 10: Filling $\frac{49}{50}$ of $T(1,1)$

$B^{4}(a-\varepsilon)$ into the torus $T(1,1)$. We describe the schematic embedding for $\varepsilon=0$. From this, an actual embedding for $\varepsilon>0$ is obtained exactly as in Example 4.10.

As in that example, given the diamond $\diamond(a)$, we decompose the square $(0,1) \times\left(-\frac{1}{2}, \frac{1}{2}\right)$ into two rectangles, and fill the right rectangle $(a-1,1) \times\left(-\frac{1}{2}, \frac{1}{2}\right)$ with the four triangles; see Figure 11(I).

The width of this rectangle is

$$
1-(a-1)=2-\sqrt{2}=: \frac{1+b}{2}, \quad \text { where } b:=3-2 \sqrt{2} .
$$

We start from a distorted diamond as in Figure 11(II), whose left flap has width $(1+b) / 2$, and so will just fit into the right rectangle. The right flap of the distorted diamond then has width $(1-b) / 2$. The height $h_{t}$ of its top triangle will be chosen 


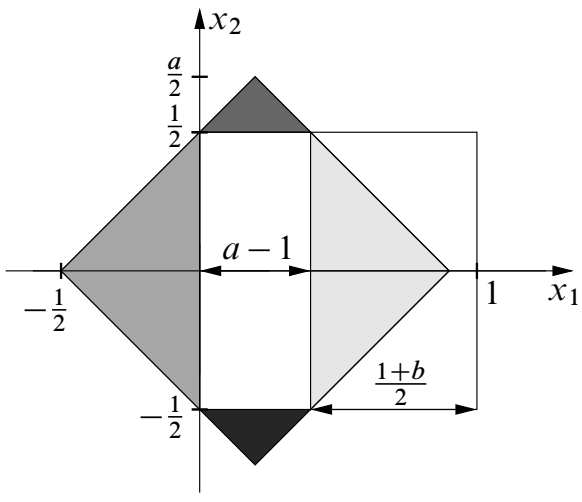

(I)

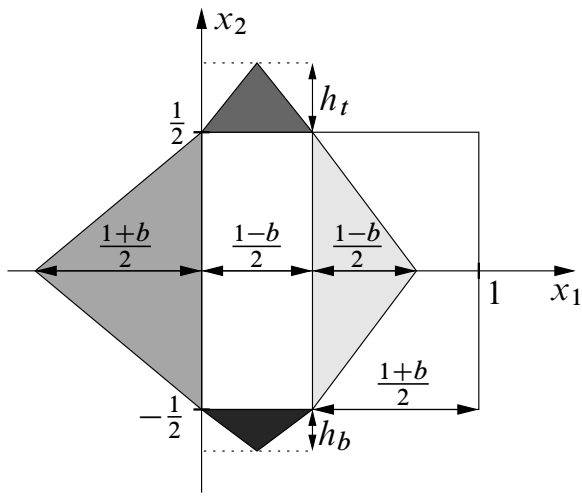

(II)

Figure 11: The decomposition of $\diamond(a)$ and its distortion

later. The height $h_{b}$ of the bottom triangle is then determined by the fact that the sum of these two heights must be $(1-b) / 2$.

Figure 12 shows the final position of the flaps in the right rectangle.

The two remaining triangles $T_{ \pm}$both have base of length $(1-b) / 2$, which agrees with that of the triangles in Figure 11(II). Therefore it remains to check that the heights $h_{t}, h_{b}$ of $T_{ \pm}$sum to $(1-b) / 2$. Note that the size of the diamond was chosen so that the total area of the triangles $T_{ \pm}$left uncovered by the flaps in Figure 12 equals the sum of the areas of the original (undistorted) top and bottom triangles. Since the base sides of $T_{ \pm}$already have the correct length $(1-b) / 2$, this equality of area forces their heights to add up to the correct amount $(1-b) / 2$ as well. In particular, these heights can be achieved by a suitable distortion.

Alternatively, one can explicitly compute the sum of $h_{t}$ and $h_{b}$ as follows. Figure 13 shows how the flaps are sheared vertically so that they fit into this rectangle. The image of the shear $\psi_{1}$ in Figure 13 (center top) shows that $h_{b}+\ell+2 b /(1+b)=1$, where $\ell=(1-3 b) /(1-b)$ is the length of the intersection of the lighter flap with the vertical line at $x_{1}=b$. Further, looking at the shear $\varphi_{2}$ we see that $h_{t}=1-(1-b) /(1+b)=$ $2 b /(1+b)$. Therefore $h_{b}+h_{t}=1-\ell=2 b /(1-b)$. Hence $h_{b}+h_{t}=(1-b) / 2$ if $2 b /(1-b)=(1-b) / 2$, or equivalently if $b^{2}-6 b+1=0$. But this holds because we defined $b=3-2 \sqrt{2}$.

In any case, as in Example 4.10, we can therefore use an $x_{1}$-shear to bring the top triangle of the distorted diamond into $T_{-}$and the bottom triangle into $T_{+}$. 


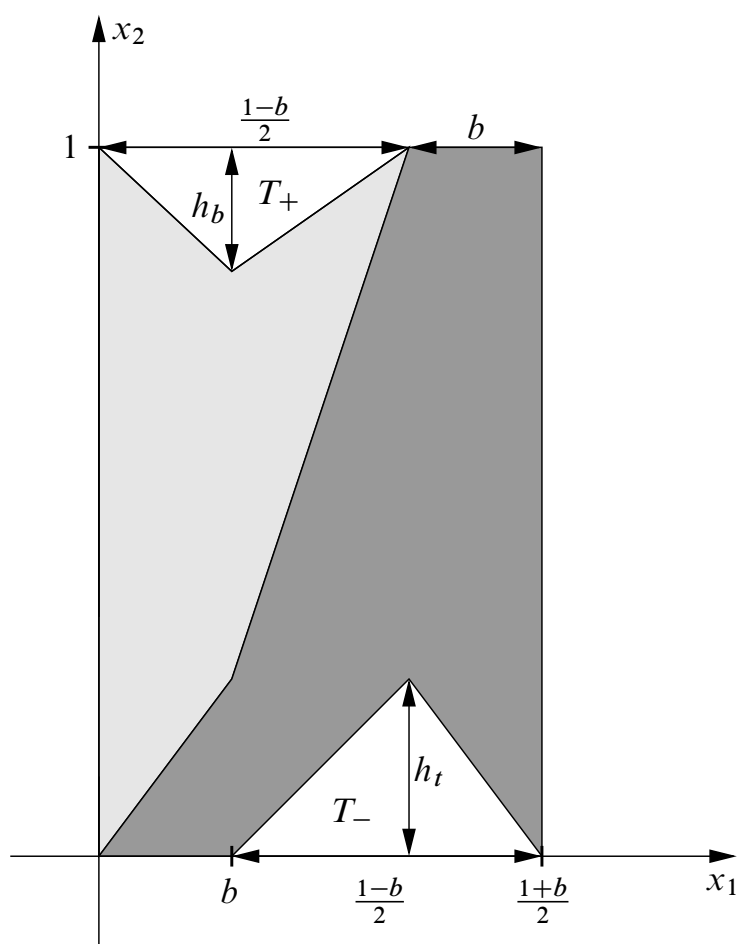

Figure 12: The final position of the flaps, where we have translated axes so that the origin lies at the corner of the right rectangle

\section{Proofs of Corollaries 1.2 and 1.3}

Proof of Corollary 1.2 Consider the 4 -torus $T^{4}$ and its blow-up $\tilde{X}=T^{4} \sharp \overline{\mathbb{C} P^{2}}$. Fix an orientation of $\tilde{X}$. Denote by $E \in H_{2}(\tilde{X} ; \mathbb{Z})$ the homology class of the exceptional divisor (with some orientation) in $\tilde{X}$. We need to show that the symplectic cone of $\tilde{X}$ is

$$
\mathcal{C}(\tilde{X})=\left\{\alpha \in H^{2}(\tilde{X} ; \mathbb{R}) \mid \alpha^{2}>0, \alpha(E) \neq 0\right\} .
$$

We first prove the inclusion $\subset$ in (6-1). The condition $\alpha^{2}>0$ holds because $\alpha$ is represented by a symplectic form compatible with the given orientation of $\tilde{X}$. The condition $\alpha(E) \neq 0$ follows from Taubes' work on the relation between Seiberg-Witten and Gromov invariants, according to which for any symplectic form $\omega$ on $\tilde{X}$ the class $E$ is representable by an embedded sphere on which $\omega$ is non-degenerate; see [38; 39].

We now prove the inclusion $\supset$ in (6-1). The projection $\pi: \tilde{X} \rightarrow T^{4}$ induces an orientation on $T^{4}$. The classes in $H^{2}(\tilde{X} ; \mathbb{R})$ can be written as $\pi^{*} \beta-a e$, where 

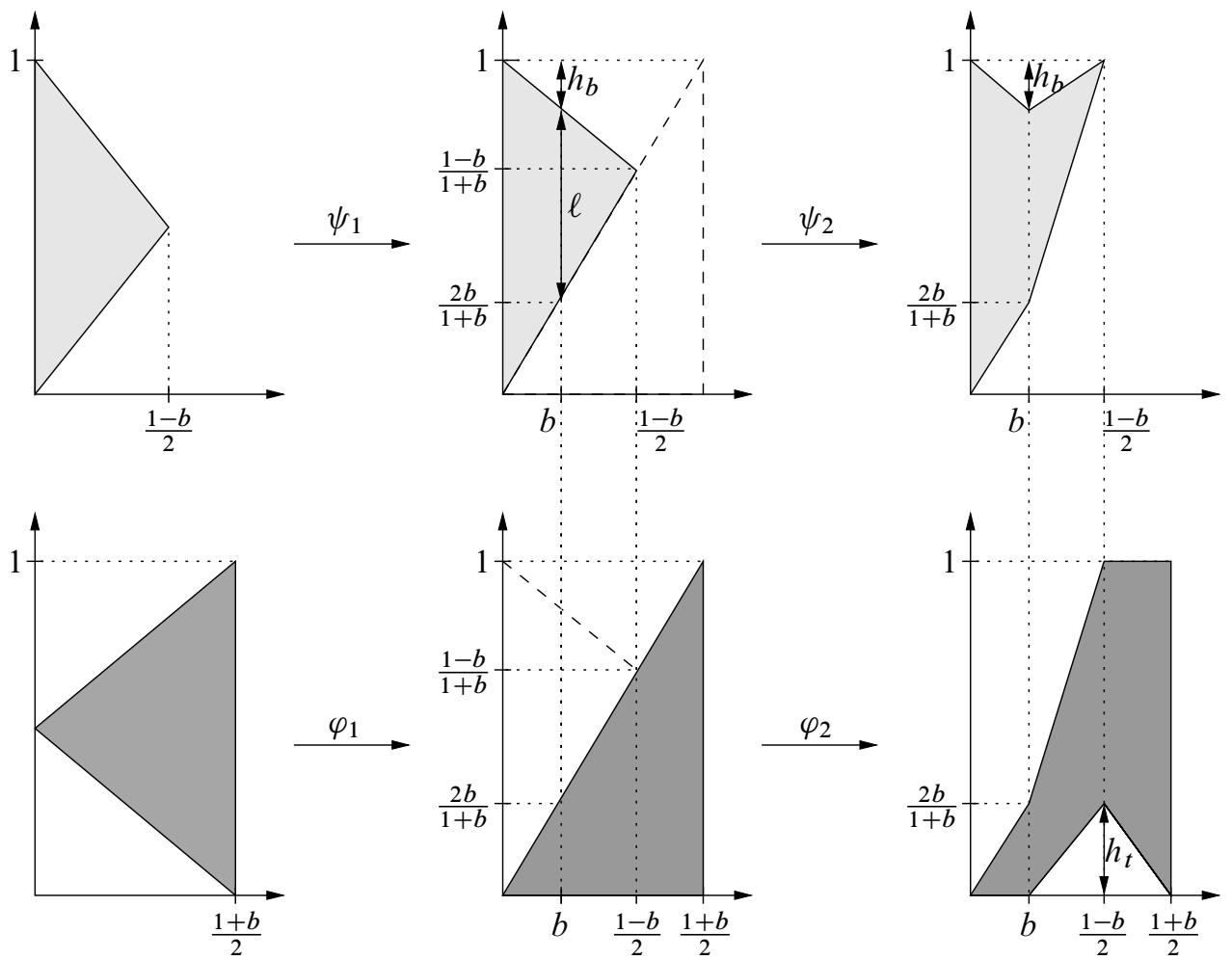

Figure 13: Filling the right rectangle with the distorted triangles. In the middle figure the darker flap has been moved down to fill half the rectangle and the lighter flap has been correspondingly sheared up. The right figure shows the effect of a further vertical shear, leaving two empty triangular regions $T_{ \pm}$, one at the top and one at the bottom.

$\beta \in H^{2}\left(T^{4} ; \mathbb{R}\right)$ and $a \in \mathbb{R}$, and where $e=\operatorname{PD}(E)$ is the Poincare dual of $E$. Since $e^{2}=-1$, the set on the right hand side of (6-1) becomes

$$
\left\{\pi^{*} \beta-a e \in H^{2}(\tilde{X} ; \mathbb{R}) \mid \beta \in H^{2}\left(T^{4} ; \mathbb{R}\right), \beta^{2}>a^{2}>0\right\} .
$$

Fix $\beta \in H^{2}\left(T^{4} ; \mathbb{R}\right)$ and $a>0$ with $\beta^{2}>a^{2}>0$. Since $\beta^{2}>0$, we can represent $\beta$ by a linear symplectic form on $T^{4}$ compatible with the given orientation. Since $\beta^{2}>a^{2}>0$, Theorem 1.1 guarantees a symplectic embedding of $B^{4}(a)$ into $\left(T^{4}, \omega\right)$. The symplectic form on the corresponding symplectic blow-up of $\left(T^{4}, \omega\right)$ represents the class $\pi^{*} \beta-a^{\prime} e$, where either $a^{\prime}=a$ or $a^{\prime}=-a$.

It is well known (see eg Wall [41, Lemma 2]) that there exists an orientation-preserving diffeomorphism $\varphi: \tilde{X} \rightarrow \tilde{X}$ of the blow-up which acts on $H^{2}(\tilde{X} ; \mathbb{R})$ by sending $e$ 
to $-e$ while fixing the orthogonal complement $\pi^{*}\left(H^{2}\left(T^{4} ; \mathbb{R}\right)\right)$ of $e$ (with respect to the cup product pairing). ${ }^{4}$ In particular, $\varphi^{*}\left(\pi^{*} \beta-a^{\prime} e\right)=\pi^{*} \beta+a^{\prime} e$. Therefore, both $\pi^{*} \beta-a e$ and $\pi^{*} \beta+$ ae belong to the symplectic cone $\mathcal{C}(\tilde{X})$.

Proof of Corollary 1.3 We denote by $\omega$ a symplectic form on $T^{4}$ such that $\left(T^{4}, \omega\right)$ is symplectomorphic to $T(1,1)$. Let again $\tilde{X}$ be the blow-up of $T^{4}$, and let $E$ be the class of the exceptional divisor $\Sigma$ (with orientation specified later). By Corollary 1.2, the class $\pi^{*}[\omega]-a \operatorname{PD}(E)$ admits a symplectic representative for all $0<a<\sqrt{2}$. We shall show that for $a>\frac{4}{3}$, this class admits no Kähler representative.

Let $\alpha$ be a Kähler form on $\tilde{X}$, and let $\pi^{*}[\omega]-a \operatorname{PD}(E)$ be its class. By the EnriquesKodaira classification, the complex manifold $(\tilde{X}, \widetilde{J})$ underlying the Kähler manifold $(\tilde{X}, \alpha)$ is the complex blow-up of a complex torus $(T, J)$. Orient $\Sigma$ by $\widetilde{J}$. The Kähler form $\alpha$ is positive on all non-constant $\widetilde{J}$-holomorphic curves in $\widetilde{X}$. In particular, $a=\int_{\Sigma} \alpha>0$, and given a non-constant $J$-holomorphic curve $C$ in $T$ with proper transform $\widetilde{C}$, we have $E \cdot[\widetilde{C}] \geq 0$ and

$$
0<\left(\pi^{*}[\omega]-a \operatorname{PD}(E)\right)([\widetilde{C}]) \leq[\omega]([C]) .
$$

The Nakai-Moishezon criterion thus implies that $[\omega]$ is an ample class on $T$. Hence $[\omega]$ gives a principal polarization of $T$. Comparing definition (2-1) with the left inequality in (6-2) we see that its Seshadri constant is at least $a$. Together with Steffens' estimate (2-5) we thus find $a \leq \frac{4}{3}$.

\section{Remarks and questions}

1 Symplectic forms on $\boldsymbol{T}^{\mathbf{4}}$ We have worked throughout with a linear symplectic form on $T^{4}$. It is not known whether every symplectic form on $T^{4}$ is isotopic to a linear form, or even whether it is symplectomorphic to such a form.

2 Very full fillings There is a stronger version of full filling: rather than asking whether one can fill an arbitrarily large fraction of the volume of a manifold $M$ with a ball, one could ask whether $M$ has a set of full measure that is symplectomorphic to an open ball. In other words, if $a=c_{G}(M, \omega)$ does the open ball $B^{4}(a)$ embed symplectically in $M$ ? Let us say that in this case $(M, \omega)$ has a very full filling (by

\footnotetext{
${ }^{4}$ Such a diffeomorphism $\varphi$ can be constructed explicitly as follows: The map $c: \overline{\mathbb{C} P^{2}} \rightarrow \overline{\mathbb{C} P^{2}}$ given by $c\left(\left[z_{0}: z_{1}: z_{2}\right]\right)=\left[\bar{z}_{0}: \bar{z}_{1}: \bar{z}_{2}\right]$ is orientation-preserving and reverses the orientation of all complex lines. By an isotopy supported near the fixed point $p_{0}=[1: 0: 0]$ we can deform $c$ to a diffeomorphism $c^{\prime}$ fixing a neighborhood of $p_{0}$, and if the connected sum to construct the blow-up is performed in this neighborhood, then $\varphi$ is obtained by glueing $c^{\prime}$ to the identity map on the torus.
} 
one ball). (There are similar versions for other filling problems.) When a rational or ruled manifold has a full filling, it also has a very full filling because one can argue as in the proof of Lemma 4.3, using the fact that in this case the space of ball embeddings is connected. However, these general arguments do not apply to tori, and it is unclear whether $T(1,1)$, for example, has a very full filling by one ball. On the other hand, the explicit fillings in Example 4.6 and Remark 4.7 give very full fillings.

3 The isotopy problem For some symplectic four-manifolds $(M, \omega)$, such as the complex projective plane or a product of 2-spheres, it is known that the space of symplectic embeddings of a given (closed) ball into $(M, \omega)$ is connected; see [30; 31]. For tori, this is a completely open problem. For many balls $B^{4}(a)$, our embedding constructions yield various symplectic embeddings into tori $T(\mu, 1)$, for which we do not know whether they are symplectically isotopic.

As a first example, consider, for some fixed small $\varepsilon>0$, the symplectic embeddings of a ball filling $\frac{8}{9}-\varepsilon$ of $T(1,1)$ that are illustrated, for $\varepsilon=0$, in Figure 14 . Here, the embedding (+-) is the one of Example 3 in Section 4.3, and the other three embeddings are obtained in the same way. Are these balls symplectically isotopic in $T(1,1)$ ? Note that, for instance, the (not Hamiltonian) symplectomorphism $\left(x_{1}, y_{1}, x_{2}, y_{2}\right) \mapsto$ $\left(-x_{1},-y_{1}, x_{2}, y_{2}\right)$ of $T(1,1)$ maps the ball $(++)$ to (--) and maps $(+-)$ to $(-+)$.

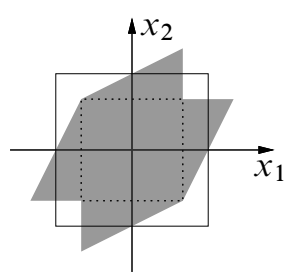

$(++)$

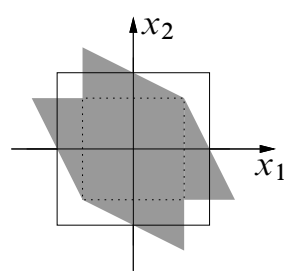

$(--)$

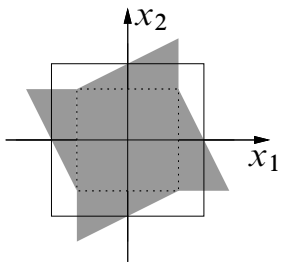

$(+-)$

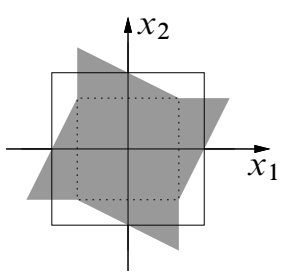

$(-+)$

Figure 14: Four embeddings of $B^{4}\left(\frac{4}{3}\right)$ into $T(1,1)$

As a second example, consider the following two full fillings of $T\left(\frac{9}{8}, 1\right)$ : The first filling is the one obtained from the explicit full filling of $T(1,72)$ via Lemma 2.1. The second filling is similar to the embedding in Example 3 of Section 4.3. We decompose the diamond $\diamond\left(\frac{3}{2}\right)$ and the rectangle $\left(0, \frac{9}{8}\right) \times(0,1)$ as in Figure $15(\mathrm{I})$, and shear the triangles as shown in Figure 15(II). ${ }^{5}$ These two embeddings are clearly different. Are they symplectically isotopic?

\footnotetext{
${ }^{5}$ This is the first in a family of full fillings of $T\left(\mu_{k}, 1\right)$ with $\mu_{k}=(2 k+1)^{2} /\left(2(k+1)^{2}\right)$ for $k \geq 1$, using the diamond $\diamond((2 k+1) /(k+1))$ in which the top triangle $x_{2} \geq \frac{1}{2}$ is sliced into $k$ horizontal slices of heights $1 /(k+1)^{2}, 2 /(k+1)^{2}, \ldots, k /(k+1)^{2}$ and then sheared to the right so that the right edge of the $j^{\text {th }}$ piece lies over an $x_{1}$-interval of length $(2 j-1) /\left(2(k+1)^{2}\right)$, while the bottom triangle is sheared symmetrically to the left.
} 

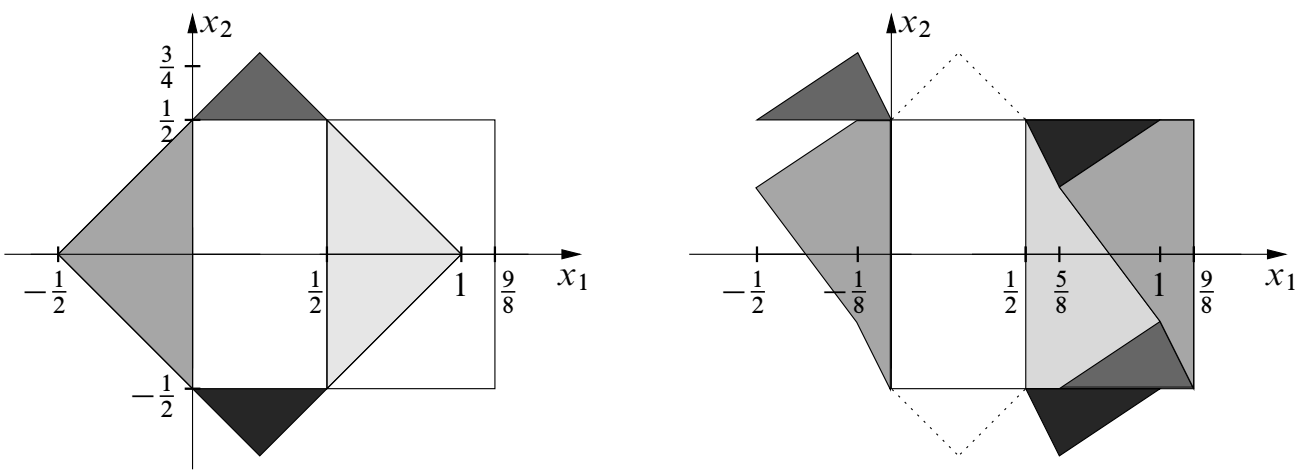

Figure 15: Another full filling of $T\left(\frac{9}{8}, 1\right)$

More generally, it is not known whether there is any $\varepsilon \in(0, \sqrt{2})$ such that the space of symplectic embeddings of $B^{4}(\varepsilon)$ into $T(1,1)$ is connected.

\section{Uniqueness of symplectic structures on the blow-up of $\boldsymbol{T}^{\mathbf{4}}$ Suppose that $\omega_{1}, \omega_{2}$} are two cohomologous symplectic forms on the blow-up of a given symplectic 4 manifold $(M, \omega)$ that are obtained by blow-up from two ball embeddings into $M$. If there is a symplectomorphism from one embedding to the other (as with the $(++),(--)$ embeddings mentioned in 3 above) then the two blow-up forms $\omega_{1}, \omega_{2}$ are diffeomorphic. However, usually there is no obvious map of this kind. Further, because the Gromov-Witten invariants of $T^{4}$ vanish, we have no tools (such as those in [30; 31]) to prove that the two blow-up forms are isotopic or even just diffeomorphic. Hence the uniqueness problem for symplectic forms on the blow-up of a 4-torus is open, even if we restrict consideration to forms on the blow-up that blow down to linear forms.

5 Higher dimensions The filling methods used in Section 3 work only in dimension 4. Although many of the explicit arguments in Section 4 extend to higher dimensions, the higher-dimensional analogs of the diamond $\diamond$ (eg, the octahedron) do not tile Euclidean space. Therefore there seem to be no simple explicit full fillings of tori by balls in higher dimensions along the lines of Example 4.6. As we explained in Section 2.3, one can get some (presumably rather weak) lower bounds for the ball filling number of tori of dimension $2 n \geq 6$ from the computations of Seshadri constants in [3] and [11]. For example, when $n=3,4$ we have:

$$
p\left(\mathrm{~T}^{6}\right) \geq \frac{288}{343} \quad \text { and } \quad p\left(\mathrm{~T}^{8}\right) \geq \frac{2}{3} .
$$

It is not clear how to do better than this, or even how to realize these bounds by explicit embeddings. It is also not clear how to find explicit embeddings in dimension 4 that do better than some of the sharper Seshadri constants, for example $\frac{360}{361}$ for $T(1,5)$. 
6 Packings by cubes and polydiscs Instead of looking at symplectic embeddings of balls, one may study symplectic embeddings of cubes and polydiscs $B^{2}\left(a_{1}\right) \times$ $\cdots \times B^{2}\left(a_{n}\right)$. (In fact, the problem of symplectically packing certain domains in $\mathbb{R}^{2 n}$ by equal cubes has been an original motivation to consider symplectic packings; see Fefferman and Phong [15], and McDuff and Polterovich [33].) This problem has been much less studied, since (to our knowledge) even in dimension four the problem of embedding a polydisc cannot be reduced to the problem of embedding a collection of balls. Ekeland-Hofer capacities [13] and the new 4-dimensional invariants from embedded contact homology (Hutchings [18]) provide obstructions for symplectic embeddings of polydiscs into certain manifolds. A few symplectic embedding constructions for polydiscs are described in [36]; eg, the shears in [36, Section 7.1] show that $T(1,1)$ can be fully filled by $B^{2}(1 / k) \times B^{2}(k)$ for each $k \in \mathbb{N}$. Similarly, the dense set of product tori $T\left(1, m^{2} / n^{2}\right)$ with $m, n \in \mathbb{N}$ relatively prime can be fully filled by a cube. Indeed, by Remark 2.2, it suffices to fill $T\left(1, k^{2}\right)$ by a cube for each $k \in \mathbb{N}$. For this, view $B^{2}(k) \times B^{2}(k)$ as $\left(-\frac{k}{2}, \frac{k}{2}\right)^{2} \times(0,1)^{2} \subset \mathbb{R}^{2}(\boldsymbol{x}) \times \mathbb{R}^{2}(\boldsymbol{y})$, and apply (similar to the map in Example 4.6) the $x_{1}$-shear defined by $\varphi\left(x_{1}, x_{2}\right)=\left(x_{1}+k x_{2}, x_{2}\right)$.

\section{References}

[1] T Bauer, Seshadri constants and periods of polarized abelian varieties, Math. Ann. 312 (1998) 607-623 MR1660259

[2] T Bauer, Seshadri constants on algebraic surfaces, Math. Ann. 313 (1999) 547-583 MR1678549

[3] T Bauer, T Szemberg, Local positivity of principally polarized abelian threefolds, J. Reine Angew. Math. 531 (2001) 191-200 MR1810121

[4] P Biran, Symplectic packing in dimension 4, Geom. Funct. Anal. 7 (1997) 420-437 MR1466333

[5] P Biran, A stability property of symplectic packing, Invent. Math. 136 (1999) 123-155 MR1681101

[6] P Biran, K Cieliebak, Symplectic topology on subcritical manifolds, Comment. Math. Helv. 76 (2001) 712-753 MR1881704

[7] C Birkenhake, H Lange, Complex abelian varieties, 2nd edition, Grundl. Math. Wissen. 302, Springer, Berlin (2004) MR2062673

[8] N Buchdahl, On compact Kähler surfaces, Ann. Inst. Fourier (Grenoble) 49 (1999) 287-302 MR1688136

[9] P Cascini, D Panov, Symplectic generic complex structures on four-manifolds with $b_{+}=1$, J. Symplectic Geom. 10 (2012) 493-502 
[10] F Catanese, K Oguiso, T Peternell, On volume-preserving complex structures on real tori, Kyoto J. Math. 50 (2010) 753-775 MR2740693

[11] O Debarre, Higher-dimensional algebraic geometry, Springer, New York (2001) MR1841091

[12] T Drăghici, The Kähler cone versus the symplectic cone, Bull. Math. Soc. Sci. Math. Roumanie 42 (1999) 41-49 MR1880654

[13] I Ekeland, H Hofer, Symplectic topology and Hamiltonian dynamics, II, Math. Z. 203 (1990) 553-567 MR1044064

[14] G Elencwajg, O Forster, Vector bundles on manifolds without divisors and a theorem on deformations, Ann. Inst. Fourier (Grenoble) 32 (1982) 25-51 MR694127

[15] C Fefferman, D H Phong, The uncertainty principle and sharp Gårding inequalities, Comm. Pure Appl. Math. 34 (1981) 285-331 MR611747

[16] M Gromov, Pseudoholomorphic curves in symplectic manifolds, Invent. Math. 82 (1985) 307-347 MR809718

[17] H Hofer, E Zehnder, Symplectic invariants and Hamiltonian dynamics, Birkhäuser, Basel (1994) MR1306732

[18] M Hutchings, Quantitative embedded contact homology, J. Differential Geom. 88 (2011) 231-266 MR2838266

[19] M-Y Jiang, Symplectic embeddings from $\mathbf{R}^{2 n}$ into some manifolds, Proc. Roy. Soc. Edinburgh Sect. A 130 (2000) 53-61 MR1742580

[20] Y Karshon, S Tolman, The Gromov width of complex Grassmannians, Algebr. Geom. Topol. 5 (2005) 911-922 MR2171798

[21] J L Lagrange, Solution d'un problème d'arithmétique, from: “Oeuvres", (J-A Serret, editor), volume 1, Georg Olms Verlag, Hildesheim (1973) 671-732

[22] F Lalonde, D McDuff, The geometry of symplectic energy, Ann. of Math. 141 (1995) 349-371 MR1324138

[23] F Lalonde, D McDuff, Hofer's $L^{\infty}$-geometry: Energy and stability of Hamiltonian flows, II, Invent. Math. 122 (1995) 35-69 MR1354953

[24] F Lalonde, D McDuff, The classification of ruled symplectic 4-manifolds, Math. Res. Lett. 3 (1996) 769-778 MR1426534

[25] A Lamari, Le cône kählérien d'une surface, J. Math. Pures Appl. 78 (1999) 249-263 MR1687094

[26] R Lazarsfeld, Lengths of periods and Seshadri constants of abelian varieties, Math. Res. Lett. 3 (1996) 439-447 MR1406008

[27] R Lazarsfeld, Positivity in algebraic geometry, I: Classical setting: line bundles and linear series, Ergeb. Math. Grenzgeb. 48, Springer, Berlin (2004) MR2095471 
[28] T-J Li, M Usher, Symplectic forms and surfaces of negative square, J. Symplectic Geom. 4 (2006) 71-91 MR2240213

[29] G Lu, Gromov-Witten invariants and pseudo symplectic capacities, Israel J. Math. 156 (2006) 1-63 MR2282367

[30] D McDuff, Blow ups and symplectic embeddings in dimension 4, Topology 30 (1991) 409-421 MR1113685

[31] D McDuff, From symplectic deformation to isotopy, from: "Topics in symplectic 4manifolds", (R J Stern, editor), First Int. Press Lect. Ser. 1, Int. Press (1998) 85-99 MR1635697

[32] D McDuff, Geometric variants of the Hofer norm, J. Symplectic Geom. 1 (2002) 197-252 MR1959582

[33] D McDuff, L Polterovich, Symplectic packings and algebraic geometry, Invent. Math. 115 (1994) 405-434 MR1262938

[34] D McDuff, D Salamon, Introduction to symplectic topology, 2nd edition, The Clarendon Press, New York (1998) MR1698616

[35] D McDuff, J Slimowitz, Hofer-Zehnder capacity and length minimizing Hamiltonian paths, Geom. Topol. 5 (2001) 799-830 MR1871405

[36] F Schlenk, Embedding problems in symplectic geometry, de Gruyter Expositions in Mathematics 40, de Gruyter (2005) MR2147307

[37] A Steffens, Remarks on Seshadri constants, Math. Z. 227 (1998) 505-510 MR1612681

[38] C H Taubes, The Seiberg-Witten and Gromov invariants, Math. Res. Lett. 2 (1995) 221-238 MR1324704

[39] C H Taubes, Seiberg Witten and Gromov invariants for symplectic 4-manifolds, First Int. Press Lect. Series 2, Int. Press, Somerville, MA (2000) MR1798809

[40] L Traynor, Symplectic packing constructions, J. Differential Geom. 41 (1995) 735-751 MR1338484

[41] C T C Wall, Diffeomorphisms of 4-manifolds, J. London Math. Soc. 39 (1964) 131-140 MR0163323

Fachbereich Mathematik, Universität Hamburg

Bundesstrasse 55, D-20146 Hamburg, Germany

Mathematics Department, Barnard College, Columbia University

MC4410, 3009 Broadway, New York, NY 10027, USA

Institut de Mathématiques, Université de Neuchâtel

Rue Emile-Argand 11, CP 158, CH-2000 Neuchâtel, Switzerland

janko.latschev@uni-hamburg.de, dmcduff@barnard.edu, schlenk@unine.ch

Proposed: Leonid Polterovich

Seconded: Danny Calegari, Yasha Eliashberg
Received: 27 September 2012 Accepted: 13 June 2013 